
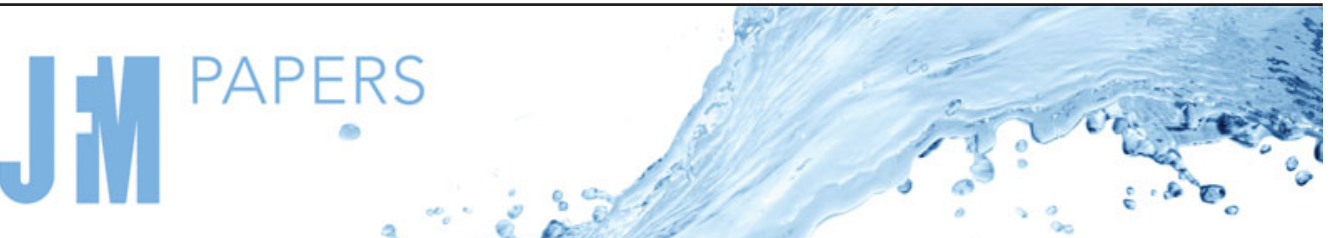

\title{
Prograde vortices, internal shear layers and the Taylor microscale in high-Reynolds-number turbulent boundary layers
}

\author{
Michael Heisel ${ }^{1,2,} \dagger$, Charitha M. de Silva ${ }^{3}$, Nicholas Hutchins ${ }^{4}$, Ivan Marusic ${ }^{4}$ \\ and Michele Guala ${ }^{1,2}$ \\ ${ }^{1}$ St. Anthony Falls Laboratory, University of Minnesota, Minneapolis, MN 55414, USA \\ ${ }^{2}$ Department of Civil, Environmental, and Geo- Engineering, University of Minnesota, Minneapolis, \\ MN 55455, USA \\ ${ }^{3}$ School of Mechanical and Manufacturing Engineering, University of New South Wales, Sydney 2052, \\ Australia \\ ${ }^{4}$ Department of Mechanical Engineering, University of Melbourne, Melbourne, Victoria 3010, Australia
}

(Received 13 October 2020; revised 22 April 2021; accepted 25 May 2021)

The statistical properties of prograde spanwise vortex cores and internal shear layers (ISLs) are evaluated for a series of high-Reynolds-number turbulent boundary layers. The considered flows span a wide range of both Reynolds number and surface roughness. In each case, the largest spanwise vortex cores in the outer layer of the boundary layer have size comparable to the Taylor microscale $\lambda_{T}$, and the azimuthal velocity of these large vortex cores is governed by the friction velocity $u_{\tau}$. The same scaling parameters describe the average thickness and velocity difference across the ISLs. The results demonstrate the importance of the local large-eddy turnover time in determining the strain rate confining the size of the vortex cores and shear layers. The relevance of the turnover time, and more generally the Taylor microscale, can be explained by a stretching mechanism involving the mutual interaction of coherent velocity structures such as uniform momentum zones with the evolving shear layers separating the structures.

Key words: boundary layer structure, turbulent boundary layers

\section{Introduction}

Similitude is one of the few properties of turbulence that makes understanding the phenomenon more tractable. This principle is indeed the basis for much of turbulence theory (Reynolds 1883; von Kármán 1930; Kolmogorov 1941; Townsend 1976).

$\dagger$ Email address for correspondence: heise070@umn.edu

(C) The Author(s), 2021. Published by Cambridge University Press. This is an Open Access article, distributed under the terms of the Creative Commons Attribution licence (http://creativecommons.org/ licenses/by/4.0/), which permits unrestricted re-use, distribution, and reproduction in any medium, provided the original work is properly cited. 


\section{Heisel, C.M. de Silva, N. Hutchins, I. Marusic and M. Guala}

The challenge, of course, is to identify the appropriate similitude (scaling) parameter for a given feature. In boundary layer research, scaling analyses have led to considerable success in characterizing the size and intensity of coherent turbulent structures (McKeon \& Sreenivasan 2007; Klewicki 2010; Marusic et al. 2010; Smits, McKeon \& Marusic 2011; Jiménez 2018). Scaling becomes particularly important for flows with high Reynolds numbers which exhibit a wider range of turbulent motions due to the increased separation between the smallest and largest scales. The friction Reynolds number $\operatorname{Re}_{\tau} \equiv u_{\tau} \delta / v$ quantifies the difference between the boundary layer thickness $\delta$ limiting the largest motions and the viscous length $v / u_{\tau}$ describing the near-wall features. Here, $v$ is the kinematic viscosity and $u_{\tau}$ is the friction velocity corresponding to the average wall shear stress.

In recent decades, the predominant focus for studies of boundary layer structures in the logarithmic (log) region has been on larger-scale, energy-containing motions (see, e.g. Marusic et al. 2010; Smits et al. 2011). In the present context, these features include both $O(\delta)$ very-large-scale motions (Kim \& Adrian 1999; Guala, Hommema \& Adrian 2006; Hutchins \& Marusic 2007) and $O(z)$ 'attached' or wall-coherent structures in accordance with Townsend's attached eddy hypothesis (Townsend 1976, p. 152), where $z$ is the wall-normal distance (see, e.g. Agostini \& Leschziner 2017; Lozano-Durán \& Bae 2019; Eich et al. 2020). While there have been numerous studies on smaller-scale vortices in the context of larger-scale features such as hairpin-type packets and vortex clusters (e.g. Adrian, Meinhart \& Tomkins 2000b; Ganapathisubramani, Longmire \& Marusic 2003; del Álamo et al. 2006; Wu \& Christensen 2006), detailed analyses of the vortex core size in boundary layer turbulence are fewer. The existing results have consistently shown the most probable vortex core diameter to be near $10 \eta$ (Tanahashi et al. 2004; Herpin, Stanislas \& Soria 2010; Herpin et al. 2013; Wei et al. 2014), where $\eta$ is the Kolmogorov length scale. The moderate discrepancy in detected size across the cited studies may be due to variations in methodology, which will be discussed further in the analysis. The characteristic velocity for the vortex, i.e. the maximum azimuthal velocity around the vortex centre, has yielded more mixed results. Tanahashi et al. (2004) argued for scaling by the Kolmogorov velocity $u_{\eta}$ despite observing moderate increase with increasing Reynolds number, and Wei et al. (2014) suggested a mixed scaling of $u_{\eta}$ and $u^{\prime}$ which is the streamwise root-mean-square (r.m.s.) velocity. The more consistent finding across studies relates to the vortex position which is less susceptible to methodology bias: the small-scale vortices are intermittently distributed and clustered in space (del Álamo et al. 2006; Wu \& Christensen 2006; Kang, Tanahashi \& Miyauchi 2007; Jiménez 2013).

The observed clustering of vortices is consistent with the apparent self-organization of the instantaneous flow in high-Reynolds-number boundary layers into large-scale velocity structures separated by relatively thin layers of concentrated shear and vorticity (Meinhart \& Adrian 1995; Priyadarshana et al. 2007; Eisma et al. 2015; de Silva et al. 2017). These thin layers are referred to here as internal shear layers (ISLs). A majority of the prograde spanwise vortex cores reside along these shear layers (Heisel et al. 2018), where prograde here indicates rotation direction consistent with the mean shear. Recent studies on ISLs have indicated robust scaling behaviour. The layer thickness is proportional to the Taylor microscale $\lambda_{T}$ (Wei et al. 2014; Eisma et al. 2015; de Silva et al. 2017) or $\delta R e_{\tau}^{-1 / 2}$ (Morris et al. 2007; Klewicki 2013), and the streamwise velocity difference across the layer is proportional to $u_{\tau}$ (de Silva et al. 2017; Heisel et al. 2018, 2020b; Gul, Elsinga \& Westerweel 2020).

The velocity structures separated by the ISLs are often identified using a general classification known as the uniform momentum zone (UMZ), a region with relatively 
uniform streamwise velocity (Meinhart \& Adrian 1995). A UMZ is uniform relative to the surrounding flow such that low-amplitude turbulent fluctuations within the zone can be neglected. UMZs are detected using histograms of the streamwise velocity (Adrian et al. $2000 b$ ) or fuzzy clustering (Fan et al. 2019), often with two-dimensional flow fields in the streamwise-wall-normal $(x-z)$ plane from imaging experiments (de Silva, Hutchins \& Marusic 2016; Saxton-Fox \& McKeon 2017; Laskari et al. 2018), including in atmospheric turbulence (Morris et al. 2007; Heisel et al. 2018). Because of their generic definition, detected UMZs are likely to be associated with other coherent velocity structures such as elongated 'streaky' structures (Hwang 2015), large-scale sweep events (Laskari et al. 2018) and travelling waves (Saxton-Fox \& McKeon 2017; Laskari \& McKeon 2021). A favourable aspect of the UMZ classification, compared with methods that detect and extract specific velocity structures localized in space, is that it allows for a systematic quantification of how the coherent velocity structures are organized throughout the boundary layer for a given measured realization of the flow. Heisel et al. (2020b) compared the UMZ properties across a wide range of Reynolds number and surface roughness to demonstrate that the organization of zones universally exhibits the theoretical scaling behaviour of the log region, i.e. size and velocity proportional to wall-normal distance and friction velocity, respectively. The findings are consistent with previous studies that showed wall-normal distance scaling of more specific isolated velocity structures such as the attached streaky structures (e.g. Hwang 2015; Hwang \& Sung 2018) and streamwise rolls (e.g. del Álamo et al. 2006; Lozano-Durán, Flores \& Jiménez 2012; Jiménez 2018).

The general self-organization of structures described above is qualitatively similar to findings in isotropic turbulence research. Specifically, shear and vortex structures are known to cluster spatially (She, Jackson \& Orszag 1990; Moisy \& Jiménez 2004). The characteristic length of the vortex tubes and the cluster is proportional to the integral length scale L (Jiménez et al. 1993; Ishihara, Gotoh \& Kaneda 2009), as is the distance between clusters (Ishihara, Kaneda \& Hunt 2013). The intense vortex tubes primarily reside within thin shear layers whose thickness is again proportional to $\lambda_{T}$ (Ishihara et al. 2013; Elsinga et al. 2017). The observations are consistent with an earlier prediction by Saffman (1968) that large-scale vortex sheets have thickness of order $\lambda_{T}$. Based on the recent findings, the organization of clustered vortex tubes into intermittent shear layers has been proposed as an important component of small-scale dynamics (Elsinga \& Marusic 2010; Hunt et al. 2010; Ishihara et al. 2013; Hunt et al. 2014; Elsinga et al. 2017). The organization is consistent with intermittency principles, where the clustering of strong velocity gradients leads to heavy-tailed probability distributions which influence high-order statistics (She \& Leveque 1994; Sreenivasan \& Antonia 1997). Despite the matched scaling and common features, there are key differences between the isotropic and boundary layer organizations. Most notably in the boundary layer case, the presence of the mean shear leads to a preferential direction and persistence of the coherent structures and significant large-scale anisotropy. Nevertheless, there is reason to be cautiously optimistic. If the behaviour of the shear layers has universal properties, it could provide a structural representation for scale interaction and the energy cascade.

In addition to the limited number of detailed studies on vortex cores and ISLs in high-Reynolds-number boundary layer turbulence, even fewer works have quantitatively explored possible relations between the coherent velocity structures, vortices and shear layers. Accordingly, the properties of both prograde spanwise vortices and internal shear layers are explored here in a single comparative analysis. The goal is to identify similarities in the behaviour of these features, and to better understand their dynamical relationship with the local larger-scale coherent velocity structures. Particular attention is 


\section{Heisel, C.M. de Silva, N. Hutchins, I. Marusic and M. Guala}

paid to why the Taylor microscale is a relevant similarity parameter for these features in high-Reynolds-number turbulence.

The behaviour of ISLs is inferred here based on the properties of detected UMZ interfaces. For the vortex cores, the analysis is focused on prograde vortices due to their closer connection with the predominately positive shear layers (i.e. $\partial u / \partial z>0$ ) that populate the boundary layer. Both analyses are conducted in the streamwise-wall-normal plane using the same suite of particle image velocimetry (PIV) experiments as in Heisel et al. (2020b), which spans a wide range of Reynolds number and surface roughness and includes a field experiment in the atmospheric surface layer (ASL, Heisel et al. 2018). The remainder of the article is organized into the following sections: the experiments and methodology are described in $\S 2$; results on the vortex and shear layer size $(\S 3)$ and velocity $(\S 4)$ are then presented; our interpretation of the results are reserved for the discussion in $\S 5$; the article concludes with a summary in $\S 6$. Two appendices are included to examine the influence of spatial resolution on the results and to justify assumptions made in the analysis.

\subsection{Terminology}

In this work, the outer layer of the boundary layer begins above the buffer layer (for smooth surfaces) or roughness sublayer (rough surfaces), and consists of the log and wake regions. The term 'small-scale' refers to the smallest turbulent eddies proportional to the Kolmogorov length $\eta$ and velocity $u_{\eta}$. The term 'large-scale' refers to the streamwise integral length $L$ and r.m.s. velocity $u^{\prime}$. In this context, the Taylor microscale is then an intermediate length scale $\lambda_{T} \sim \eta^{2 / 3} L^{1 / 3}$ (Pope 2000), where ' $\sim$ ' means 'scales with'. The term 'vortex core' is used here to differentiate the detected strongly rotating vortices from larger-scale, diffuse vortical motions such as rolls and bulges. The estimated probability density function (p.d.f.) of any variable ' $s$ ' is given the notation $p_{s}$. Unless otherwise noted, lowercase lettering is used for instantaneous values of a variable, and uppercase lettering is used for the average of the same variable. Angled brackets ' $\langle\cdot\rangle$ ' are also used to indicate ensemble averages. Bold typeface is used for variables that are vectors. The superscript ' + ' indicates normalization in wall units, i.e. $z^{+}=z u_{\tau} / v$ and $u^{+}=u / u_{\tau}$. The subscript ' $\omega$ ' is used for variables corresponding to vortex properties. The subscript ' $i$ ' is used for variables corresponding to ISL properties. Finally, as stated above, the coordinate system employed here uses $z(w)$ for the wall-normal direction (velocity).

\section{Methodology}

\subsection{Experiments}

Nine flow cases covering several orders of magnitude in Reynolds number $\operatorname{Re}_{\tau} \sim O\left(10^{3}-\right.$ $\left.10^{6}\right)$ and surface roughness $k_{s}^{+} \lesssim O\left(10^{4}\right)$ are included in the present analysis. Here, $k_{s}$ is the equivalent sand-grain roughness and conditions are fully rough for $k_{s}^{+} \gtrsim 70$ when the surface drag becomes independent of viscosity. The flow cases, previously used together in Heisel et al. (2020b), are summarized in table 1. An overview of important details is given here, and a full account of each experiment is available from the cited references in the table.

The direct numerical simulation (DNS) of a boundary layer with $R e_{\tau}=2000$ by Sillero et al. (2013) is included for comparison with the experimental cases. The DNS case was analysed using two-dimensional flow fields in the $x-z$ plane to be consistent with the PIV experiments. The variable $\Delta x$, representing the spatial resolution, refers to the streamwise 
Vortices and shear layers in turbulent boundary layers

\begin{tabular}{|c|c|c|c|c|c|c|c|c|}
\hline Dataset & Symbol & Facility & $R e_{\tau}$ & $k_{s}^{+}$ & $\Delta x^{+}$ & $\Delta x / \eta$ & $\Delta x / \lambda_{T}$ & Source \\
\hline DNS & * & computation & 2000 & - & 6.3 & 2.0 & 0.077 & $\begin{array}{l}\text { Sillero, Jiménez \& } \\
\text { Moser (2013) }\end{array}$ \\
\hline Smooth wall & $x$ & SAFL & 3800 & - & 13 & 3.3 & 0.10 & Heisel et al. $(2020 b)$ \\
\hline Smooth wall & + & SAFL & 4700 & - & 18 & 4.2 & 0.12 & Heisel et al. $(2020 b)$ \\
\hline Smooth wall & 0 & HRNBLWT & 6600 & - & 3.7 & 0.86 & 0.023 & Squire et al. (2016) \\
\hline Smooth wall & $\square$ & HRNBLWT & 12300 & - & 7.2 & 1.4 & 0.030 & Squire et al. (2016) \\
\hline Smooth wall & $\diamond$ & HRNBLWT & 17000 & - & 10 & 1.8 & 0.037 & Squire et al. (2016) \\
\hline Mesh roughness & $\Delta$ & SAFL & 10100 & 430 & 19 & 3.9 & 0.10 & Heisel et al. (2020b) \\
\hline Mesh roughness & $\nabla$ & SAFL & 13900 & 620 & 28 & 5.2 & 0.12 & Heisel et al. (2020b) \\
\hline ASL & 0 & Eolos & $O\left(10^{6}\right)$ & 30000 & 6400 & 410 & 3.2 & Heisel et al. (2018) \\
\hline
\end{tabular}

Table 1. Experimental datasets used in the analysis of prograde spanwise vortices and ISLs.

grid spacing for the DNS and the vector field spacing for the PIV experiments, which all employed $50 \%$ window overlap leading to interrogation window size $2 \Delta x$. The parameters $\eta$ and $\lambda_{T}$ are both dependent on wall-normal distance $z$. The normalized resolution in table 1 considers the average parameter values at $z=0.1 \delta$. This reference position was chosen in terms of $\delta$ because later results are presented at matched $z / \delta$ in the outer layer. Determination of $\eta$ and $\lambda_{T}$ is detailed in $\S 2.2$.

The HRNBLWT facility in table 1 refers to the High Reynolds Number Boundary Layer Wind Tunnel at the University of Melbourne. The flow cases corresponding to the HRNBLWT were measured using the high-resolution tower PIV configuration detailed in Squire et al. (2016). The sandpaper roughness flow cases from the same study are not included in the present analysis. In this case a higher degree of small-scale noise in the rough-wall measurements (as documented in Squire et al. 2016) precludes the possibility of accurately fitting the Oseen vortex model to extract vortex statistics, and hence these data are omitted from the present study.

The SAFL facility in table 1 is the boundary layer wind tunnel at St. Anthony Falls Laboratory, University of Minnesota. These measurements captured the bottom portion of the boundary layer thickness, including the log region and part of the wake region. For the mesh roughness cases, the tunnel floor was covered by woven wire mesh with $3 \mathrm{~mm}$ wire diameter and $25 \mathrm{~mm}$ opening size (Heisel et al. 2020b).

The ASL measurements were acquired using super-large-scale PIV (SLPIV) in the lowest $20 \mathrm{~m}$ of the atmosphere with natural snowfall as the flow tracers. The measurements captured the roughness sublayer and bottom of the log region in near-neutral conditions at the Eolos field facility. A detailed discussion of the snowflake traceability is given in Heisel et al. (2018). The SLPIV method cannot capture the smallest vortical motions in the ASL due to both the non-negligible snowflake inertia and the coarse spatial resolution relative to $\eta$. Yet numerous vortices were present in each SLPIV vector field with size $O(1 \mathrm{~m})$ likely augmented by coarse resolution. The vortex and ISL size statistics are included in this study, but caution must be taken in interpreting these results. Vortex and shear layer velocity statistics in the ASL, however, are shown to be in agreement with all laboratory-scale trends.

Profiles of the first- and second-order velocity statistics are shown for each flow case in figure 1 . For two of the smooth-wall cases $(\times,+)$, the relatively lower wall-normal velocity statistics in figure $1(c, d)$ are attributed to measurement resolution as discussed in Heisel et al. $(2020 a)$. The decreasing trends in figure $1(c, d)$ for the ASL case $(\bullet)$ may be due to one or more of several challenges present in atmospheric field settings. For instance, 

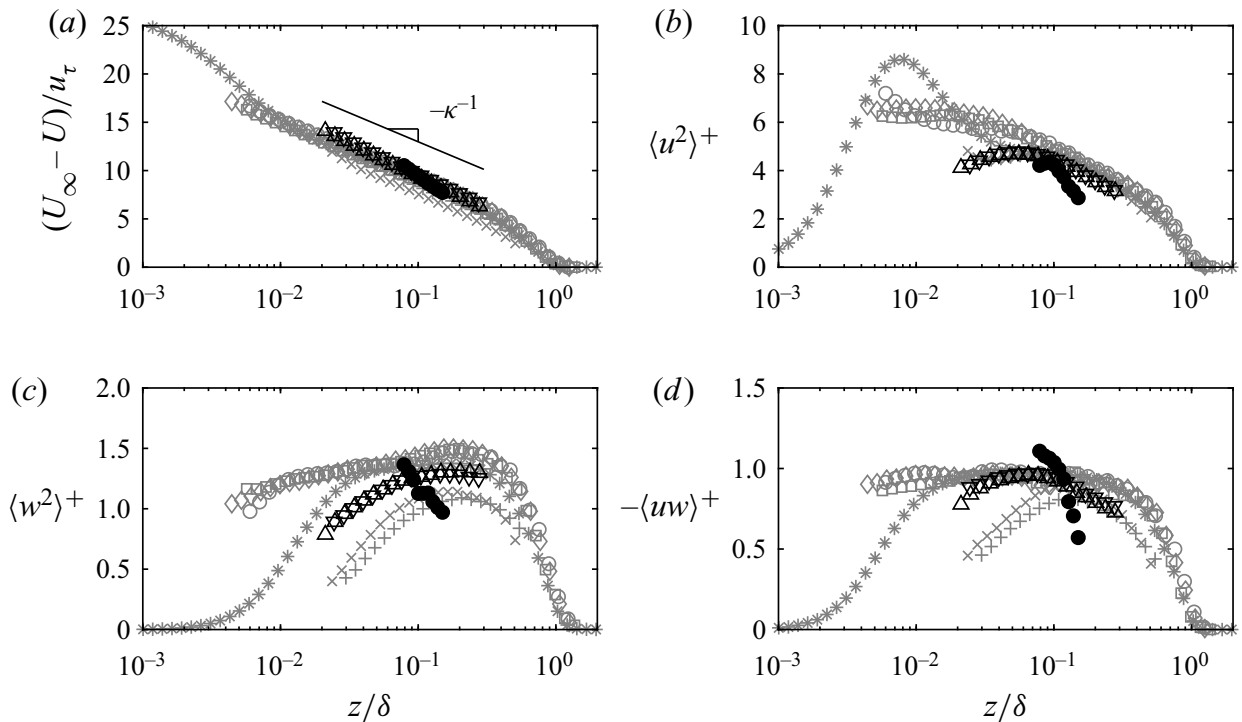

Figure 1. First- and second-order velocity statistics for each experimental dataset. (a) Mean streamwise velocity $U$ shown as a deficit from the free-stream condition $U_{\infty}$, where $\kappa^{-1}$ is the logarithmic slope based on the von Kármán constant. (b) Streamwise variance $\left\langle u^{2}\right\rangle$. (c) Wall-normal variance $\left\langle w^{2}\right\rangle$. (d) Reynolds shear stress $-\langle u w\rangle$. Profiles are normalized by the friction velocity $u_{\tau}$ and boundary layer thickness $\delta$. Data symbols correspond to the experiments in table 1 and are shown with logarithmic spacing for clarity. The roughness sublayer is excluded from the ASL profiles.

uncertainty in the definition of $\delta$, statistical convergence of higher-order statistics, and the possible role of large-scale stratified motions at higher altitudes are all discussed in the original study (Heisel et al. 2018).

\subsection{Scaling parameters}

Owing to their importance in the scaling analysis, the determination of relevant flow parameters is discussed here. Not included here is $u_{\tau}$, which is discussed in the original publications cited in table 1. For the wind tunnel studies (HRNBLWT and SAFL), the parameters were estimated using hot-wire anemometer measurements under the same flow conditions rather than the PIV measurements (Squire et al. 2016; Heisel et al. 2020b). Parameter estimation for the ASL case relied on scaling assumptions which are discussed separately at the end of this section.

Many of the parameters depend on the average rate of turbulent energy dissipation $\epsilon$. The Kolmogorov scales are defined as the length $\eta \equiv\left(v^{3} / \epsilon\right)^{1 / 4}$, velocity $u_{\eta} \equiv(v \epsilon)^{1 / 4}$ and time $\tau_{\eta} \equiv(v / \epsilon)^{1 / 2}$. Further, the definition used here for the streamwise integral length is $L=u^{\prime 3} / \epsilon$ and for the large-eddy turnover time is $T=u^{\prime 2} / \epsilon$. This integral length definition yields results similar to the autocorrelation method, based on a test using the hot-wire measurements. The turnover time $T$ is equivalent to the integral time scale definition in isotropic turbulence where $T=L / u^{\prime}$, and differs from the streamwise integral time in wall-bounded flows where $T_{\text {int }}=L / U$.

Because $\epsilon$ could not be measured directly, various estimation methods were used depending on the experiment. Dissipation was estimated as $\epsilon \approx 15 v\left\langle(\partial u / \partial x)^{2}\right\rangle$ assuming local isotropy with the HRNBLWT measurements (Squire et al. 2016). For the DNS and SAFL cases, the dissipation was inferred from the value of the longitudinal second-order 
structure function in the inertial subrange, i.e. $D_{11}=C \epsilon^{2 / 3} r^{2 / 3}$ (Saddoughi \& Veeravalli 1994).

The Taylor microscale is defined here as $\lambda_{T}^{2}=u^{\prime 2} /\left\langle(\partial u / \partial x)^{2}\right\rangle$ and the value was estimated using this expression for all cases excluding the ASL. This definition is specific to the streamwise component, where the Taylor microscale is anisotropic in wall-bounded turbulence (Morishita, Ishihara \& Kaneda 2019). The Taylor microscale and integral length are related as $\lambda_{T} / L \sim R e_{L}^{-1 / 2}$ with the integral Reynolds number being $R e_{L}=u^{\prime} L / v$ (Pope 2000). This relationship is based on $\epsilon \sim \nu\left\langle(\partial u / \partial x)^{2}\right\rangle$, where the proportional constant is 15 for isotropic turbulence and otherwise depends on the relative contribution of each gradient term to the dissipation.

For the ASL case, the measurement resolution of both the SLPIV and a collocated sonic anemometer were too coarse for the estimation methods discussed above. The dissipation was therefore estimated as $\epsilon \approx u_{\tau}^{3} / \kappa z$, where $\kappa$ is the von Kármán constant. This estimate assumes the local shear production of turbulence in the log region is in equilibrium with the dissipation rate, which is typical for high-Reynolds-number boundary layers (Townsend 1961). The Kolmogorov length scale resulting from this approximation is $\eta \approx 0.7 \pm 0.2 \mathrm{~mm}$. The value for $\lambda_{T}$ was estimated from the expression $\epsilon=15 v u^{\prime 2} / \lambda_{T}^{2}$, which assumes local isotropy and combines the $\lambda_{T}$ and $\epsilon$ expressions through the shared gradient term. The resulting Taylor microscale estimate for the ASL case is $\lambda_{T} \approx 13 \mathrm{~cm}$. The integral length $L \sim O(1 \mathrm{~m})$ results directly from the estimated dissipation.

\subsection{Spanwise vortex detection}

Numerous methods exist to characterize vortices (see, e.g. Chakraborty, Balachandar \& Adrian 2005; Haller 2005). The preferred method in the present analysis is to fit the local flow field to a model vortex. The primary advantage of using a model vortex is that the fitted vortex properties are not dependent on an arbitrary parameter threshold. However, a parameter threshold is still required to detect the possible vortex core regions upon which the model is fitted, and the characterization of complex vortex structures is limited to the imposed model shape. Despite these limitations, recent studies have successfully characterized large populations of vortex cores in boundary layer flows using the Oseen model (Oseen 1912) in cylindrical coordinates (Carlier \& Stanislas 2005; Herpin et al. 2010, 2013)

$$
\boldsymbol{u}=\boldsymbol{u}_{\omega}+\frac{\Gamma}{2 \pi r}\left[1-\exp \left(-\left(\frac{r}{r_{\omega}}\right)^{2}\right)\right] \boldsymbol{e}_{\theta},
$$

where $\boldsymbol{u}_{\omega}$ is the advection velocity of the vortex, $\Gamma$ is the circulation, $r$ is the radial distance from the vortex centre, $r_{\omega}$ is the vortex radius and $\boldsymbol{e}_{\theta}$ is the unit vector in the azimuthal direction. The model incorporates Biot-Savart law for the velocity induced by a vortex line, i.e. $u_{\theta} \propto \Gamma / 2 \pi r$, and the exponential term acts as a damping function to decrease the azimuthal velocity $u_{\theta}$ inside the vortex radius such that the maximum velocity occurs at $r=r_{\omega}$.

The Oseen model is consistent with the Navier-Stokes equations in its three-dimensional definition. In the formal definition, the radius is given as $r_{\omega}=\sqrt{4 v t}$, where the radius is zero at time $t=0$ and the vortex grows in time via viscous diffusion. This is conceptually different from the Burgers vortex radius $r_{\omega}=\sqrt{4 v / \alpha}$, where $\alpha$ is the strain rate. Rather than growing in time, the Burgers vortex size is steady due to a balance between outward diffusion and an inward radial velocity $u_{r}(r)=-\alpha r$ induced by axial vortex stretching (Burgers 1948). The Oseen model does not include a radial velocity or axial stretching. 


\section{Heisel, C.M. de Silva, N. Hutchins, I. Marusic and M. Guala}

This distinction between the two models becomes important for interpreting the later results.

The vortex detection using (2.1) followed the procedure of Herpin et al. (2013), which is briefly summarized here. Possible vortex cores were identified based on flow regions with $\lambda_{c i}>1.5 \lambda_{r m s}$, where $\lambda_{c i}$ is the two-dimensional swirling strength (Adrian, Christensen $\&$ Liu 2000a) and $\lambda_{r m s}(z)$ is the r.m.s. swirling strength at each wall-normal distance. The threshold introduces selection bias on the vortices, but the threshold is not applied in the later model fit. Based on a test using one flow case, changing the threshold factor from $1.5 \times$ to $2 \times$ or $2.5 \times$ resulted in the detection of fewer vortices, but resulted in no statistically significant changes to the vortex properties.

A notable deviation to the original procedure was to apply a Gaussian filter to the wind tunnel (HRNBLWT and SAFL) PIV fields prior to vortex detection. The filter removed small-scale noise in the gradients which significantly improved the performance of the fitting algorithm. The size of the Gaussian filter, i.e. its standard deviation, was selected to be approximately $2 \eta$ for the reference position $z=0.1 \delta$ in each case. The filter was not applied to the DNS or SLPIV vector fields. The filter would be negligibly small relative to the resolution for the ASL case, and a comparison of DNS results with and without the filter revealed no changes to the statistics of interest.

The properties of the identified core region were used as initial guesses for the six vortex model parameters: the centre position given by $x_{\omega}$ and $z_{\omega}$, the advection velocity components $u_{\omega}$ and $w_{\omega}$, the circulation $\Gamma$ and the radius $r_{\omega}$. The initial value for $\Gamma$ was based on the core area and average out-of-plane vorticity $\omega_{y}$ within the core region. The initial guesses for $r_{\omega}, x_{\omega}$ and $z_{\omega}$ were used to extract the local vector field within $2 r_{\omega}$ of the vortex centre. The extracted vector field was then fitted to (2.1) using the initial guesses and a nonlinear least-squares fitting algorithm. The algorithm was designed to update the six model parameters until the difference between the local vector field and the model vortex (i.e. the right-hand side of (2.1)) was minimized. The parameters output by the algorithm therefore describe the model vortex most closely matching the vector field within and around the vortex core. The parameters are thus inferred to represent the vortex core properties. The initial guesses informed by the detected core regions reduce the likelihood the algorithm diverged to a spurious local minimum in the model parameter space. To exclude these fits and other ill-fitted results, the properties are only considered for vortices where the coefficient of determination exceeded $R^{2}>0.6$. Increasing the imposed minimum $R^{2}$ value reduces somewhat the convergence of the vortex statistics, but does not change the overall trends and conclusions of the study. An example vector field and closest model fit with $R^{2}=0.9$ are shown in figure 2(a).

Besides the advection velocity, the characteristic velocity of each vortex is the maximum azimuthal velocity difference $\Delta u_{\omega}$ across the vortex. This velocity, shown in figure $2(b)$, is twice the azimuthal velocity at the edge of the vortex $r=r_{\omega}$ due to axisymmetry and the definition of the radius. From (2.1), the velocity is

$$
\Delta u_{\omega}=\frac{\Gamma}{\pi r_{\omega}}\left(1-e^{-1}\right) .
$$

The properties are defined across both sides of the vortex core, i.e. as $d_{\omega}$ and $\Delta u_{\omega}$, for comparison with the ISL properties which are not in cylindrical coordinates.

The detection algorithm was conducted for all experimental datasets in table 1 . Approximately 22000 Oseen vortices were fitted for the ASL dataset, and more than 100000 were fitted for every other case. The possible issue of selection bias due to the chosen vortex model is discussed later in $\$ 5.3$. The primary sources of uncertainty for 
(a)

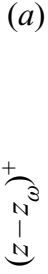

(b)

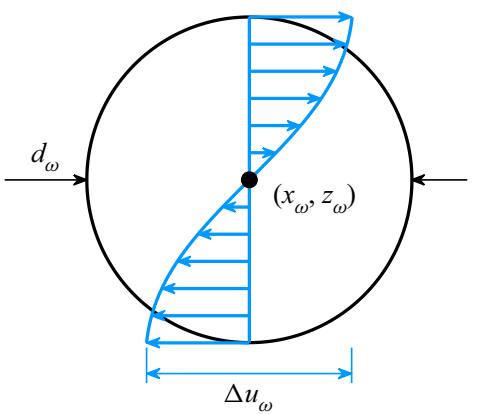

Figure 2. Example of a model Oseen vortex fitted to a velocity vector field, shown with the vortex advection velocity subtracted for visualization. (a) Comparison of measured velocities (black vectors) relative to the closest Oseen model (red vectors) whose size is given by the red circle. (b) Notation for the diameter $d_{\omega}$ and azimuthal velocity difference $\Delta u_{\omega}$ across the diameter of the fitted vortex.

vortex properties in the PIV experiments are spatial resolution limitations and small-scale noise. The results are focused on probability distributions of the properties rather than their mean. Artefacts due to the limitations described above occur primarily within the smallest values of each property, and conclusions are not drawn from these regions of the distributions. See Appendix A for an assessment of how measurement resolution affects the mode and tail of the probability distributions. A further benefit of a probabilistic approach is to identify the possible relevance of multiple scaling parameters. If different regions of the probability distribution are governed by separate parameters, the mean value may not reflect a simple scaling relationship. A similar probability analysis of the ISL features is unfortunately not possible for the methodology given in the following section. The analysis requires conditionally averaging which does not provide instantaneous statistics.

\subsection{ISL detection}

Rather than directly detecting the ISLs using a threshold on instantaneous values of $\partial u / \partial z$, we instead infer the properties of ISLs from detected interfaces of UMZs. These interfaces represent the shear regions separating the local larger-scale coherent velocity structures (de Silva et al. 2017). In the present context, the distinction between ISLs and UMZ interfaces is largely a matter of detection methodology. While the shear layers align closely with UMZ interfaces, the interfaces can also include segments where the instantaneous shear is relatively smaller (Gul et al. 2020). These additional segments due to the detection methodology may lead to differences between ISL and UMZ interface statistics for some properties. However, the statistics presented here are limited to those where the same behaviour has been previously reported for both ISLs and UMZ interfaces, and from here on the ISL terminology is applied.

The present analysis uses the same detected UMZs from Heisel et al. (2020b). The methodology to identify UMZs and their interfaces using histograms of the streamwise velocity is detailed in the previous work. An important consideration regarding the previous study is that the detected UMZs unambiguously exhibited a dependence on wall-normal distance $z$ (Heisel et al. 2020b), such that the present analysis studies the shear layers separating $z$-scaled velocity structures. 

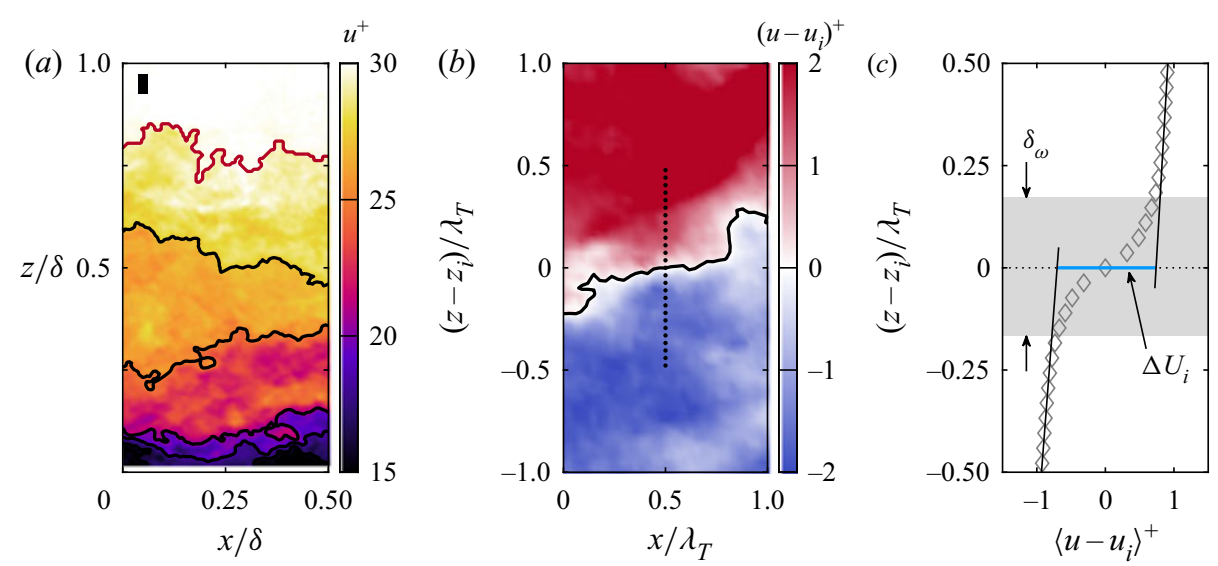

Figure 3. Example shear layers for the $R e_{\tau}=17000$ smooth-wall case. (a) Streamwise velocity field from figure 2 of Heisel et al. (2020b), with detected ISLs (black lines) and turbulent/non-turbulent interface (red line). (b) Velocity field in the vicinity of an ISL (black line) relative to the ISL position $z_{i}$ and velocity $u_{i}$. $(c)$ Conditionally averaged velocity profile relative to the ISLs, indicating the average velocity difference $\Delta U_{i}$ and thickness $\delta_{\omega}$. The black box in the upper left of $(a)$ indicates the size of the field in $(b)$.

Figure 3 shows an example of ISLs and their statistics based on measurements from the $R e_{\tau}=17000$ smooth-wall case in table 1. The PIV field in figure 3(a) illustrates the general, approximate organization of the streamwise velocity in the outer layer into relatively uniform regions (i.e. UMZs) separated by thin shear layers (black lines). The field in figure $3(b)$ is focused on the local vicinity of a single ISL to demonstrate the compiled statistics. The velocity difference between UMZs is a factor of $u_{\tau}$, and the velocity gradient occurs across a short distance centred around the wall-normal position $z_{i}$ of the ISL. The black dots in figure 3(b) represent the PIV vector positions near the ISL at the horizontal centre of the field. The PIV velocities were compiled at these vector positions to create a profile relative to the ISL position $\left(z-z_{i}\right)$ and velocity $\left(u-u_{i}\right.$ and $w-w_{i}$ ). These conditional profiles were compiled for every $x$ position of every ISL.

Averaging all the conditional streamwise profiles in the log region yields the profile in figure $3(c)$. As seen in the figure, the average velocity difference $\Delta U_{i}$ results from linear fits to the profile above and below the ISL (de Silva et al. 2017). One method for estimating the (vorticity) thickness $\delta_{\omega}$ is to assume the ISL is a mixing layer (Brown \& Roshko 1974). Note the subscript ' $\omega$ ' is used for the shear layer thickness $\delta_{\omega}$ to be consistent with notation in the literature. The mixing layer method uses the maximum velocity gradient within the layer $\partial\left\langle u-u_{i}\right\rangle /\left.\partial z\right|_{\max }$. The gradient is highest at the centre of the layer and quickly decreases away from the centre, such that the maximum gradient is not representative of the entire layer. In practice, the measured maximum gradient also depends strongly on the spacing of the grid points $\Delta x$ in the vicinity of the layer centre, and $\delta_{\omega}$ values from this method are a function of the measurement resolution.

The thickness $\delta_{\omega}$ can more simply be estimated as the distance across which the velocity difference $\Delta U_{i}$ occurs (Eisma et al. 2015). In addition to being less sensitive to measurement resolution, this estimate is more representative of the overall shear layer. This second method is used to calculate the thicknesses presented in later results. Note this method is still susceptible to resolution issues if the measurements are coarse relative to $\delta_{\omega}$, as is discussed later. 

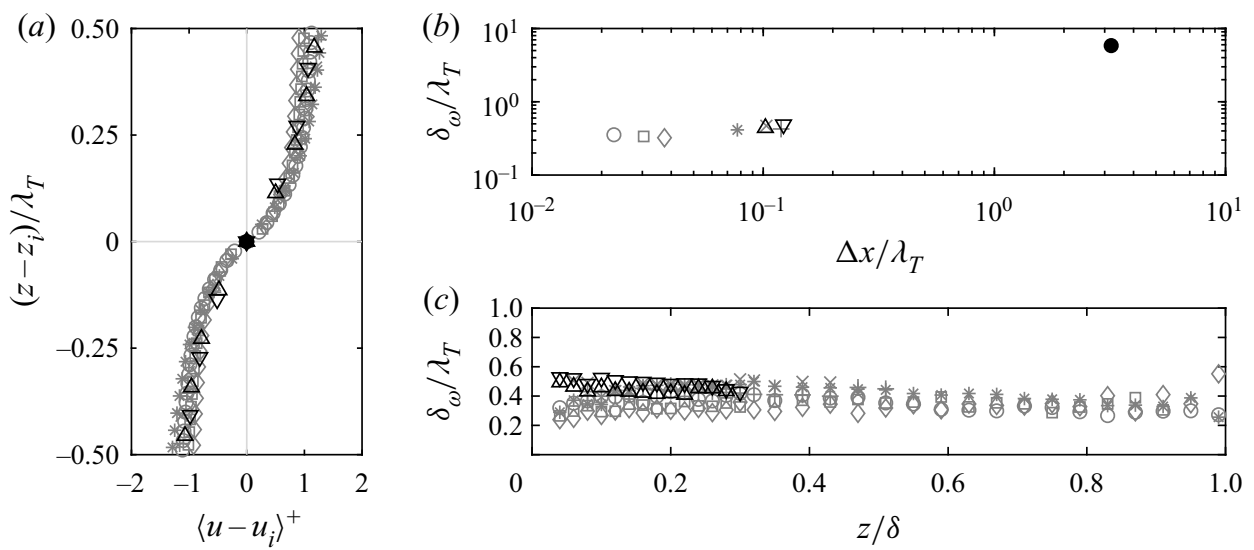

Figure 4. Estimated average thickness $\delta_{\omega}$ of ISLs normalized by $\lambda_{T}$. (a) Conditional average profile of streamwise velocity relative to ISLs in the log region. (b) Thickness $\delta_{\omega}$ as a function of measurement grid spacing $\Delta x$, noting the interrogation window size for the PIV cases is $2 \Delta x$. (c) Wall-normal profile of $\delta_{\omega}$. Data symbols correspond to the experimental datasets in table 1.

In many respects, it is important to distinguish the ISLs from the turbulent/non-turbulent interface (TNTI) separating the boundary layer flow from the free-stream condition (Elsinga \& da Silva 2019), i.e. the red line in figure 3(a). Indeed, the TNTI was detected using a separate methodology based on the kinetic energy defect (Chauhan et al. 2014b) prior to detection of the UMZs and the internal layers (Heisel et al. 2020b). However, for the specific statistics analysed in the present study, the same scaling behaviour is expected for both ISLs and the TNTI. Previous studies have shown the TNTI to have thickness $\delta_{\omega} \sim O\left(\lambda_{T}\right)$ and velocity $\Delta U \sim O\left(u_{\tau}\right)$ (Bisset, Hunt \& Rogers 2002; da Silva \& Taveira 2010; Chauhan, Philip \& Marusic 2014a; de Silva et al. 2017), matching the ISL literature results discussed in the introduction. Later figures showing wall-normal profiles of shear layer properties include ISLs and TNTIs, such that both features contribute to values in the wake region.

\section{Size statistics}

\subsection{Shear layer thickness}

The streamwise velocity profile relative to the ISLs, shown previously in the figure $3(c)$ example, is compared for all flow cases in figure 4(a). The ASL measurement resolution exceeds the vertical axis limits and the profile is not visible. There is otherwise close agreement across cases with normalization by $\lambda_{T}$ and $u_{\tau}$. A comparison of the profiles with alternative length scale normalizations, e.g. $v / u_{\tau}$ and $\eta$, is presented elsewhere (e.g. de Silva et al. 2017).

The average ISL thickness was calculated from the conditional profiles following the methodology discussed in $\$ 2.4$. The effect of measurement resolution on the estimated thickness is demonstrated in figure $4(b)$. The thickness estimate is relatively invariant to resolution for grid spacing $\Delta x \lesssim 0.1 \lambda_{T}$. The four SAFL PIV cases with $\Delta x \approx 0.1 \lambda_{T}$ have mean thickness approximately $25 \%$ larger than the cases with smaller $\Delta x$, suggesting the effects of resolution may begin near this value. Assuming the $\lambda_{T}$ scaling extends to field conditions, the resolution of the ASL measurements is too coarse for an accurate estimate of the ISL thickness, and no conclusions are drawn from the $\delta_{\omega}$ value in the ASL case. 


\section{Heisel, C.M. de Silva, N. Hutchins, I. Marusic and M. Guala}

A wall-normal profile of $\delta_{\omega}$ was constructed using conditional ISL statistics in binned intervals of $z / \delta$. The resulting profile in figure $4(c)$ suggests a fixed relationship between $\delta_{\omega}$ and $\lambda_{T}$ throughout the outer layer. The proportionality $\delta_{\omega} \approx(0.3-0.5) \lambda_{T}$ and its invariance with wall-normal position are both in close agreement with previous studies (Eisma et al. 2015; de Silva et al. 2017). Note that normalization of $\delta_{\omega}$ by $\delta e_{\tau}^{-1 / 2}$ collapses the profiles across experiments, but the thickness varies with $z$, i.e. the profiles are not flat. The local parameter $\lambda_{T}(z)$ is required to achieve the observed invariance of $\delta_{\omega}(z)$ with wall-normal position. The scatter between cases in figure $4(c)$ is entirely consistent with the resolution findings in figure $4(b)$ : the cases with coarser resolution relative to $\lambda_{T}$ have profile values closer to $0.5 \lambda_{T}$, and cases with higher resolution have profile values closer to $0.3 \lambda_{T}$. A new finding from the present results is that significant laboratory-scale roughness (i.e. $k_{s}^{+} \approx 500$ ) does not modify the relationship between $\delta_{\omega}$ and $\lambda_{T}$ beyond the uncertainty due to resolution, thus confirming the scaling is not limited to smooth-wall conditions. Ebner, Mehdi \& Klewicki (2016) proposed that large roughness may affect the thickness of the shear layers and the overall organization of UMZs. However, the required roughness in terms of $k_{s} / \delta$ for this effect to occur may be too great to maintain outer layer similarity.

\subsection{Vortex diameter}

As discussed in $\$ 2.3$, the prograde spanwise vortex core statistics are presented here as probability distributions rather than averages. Specifically, statistics are given as p.d.f.s, which were estimated discretely as binned histograms with amplitude scaled to achieve area unity, i.e. probability of one within the parameter space. To improve the statistical convergence of the probability tails, logarithmic spacing was used for the histogram binning intervals. The proper normalization of the histogram as a p.d.f. is then achieved by dividing the value of each bin by its respective bin width and the total number of occurrences in the histogram. Logarithmic bin intervals were only used where the scale of the abscissa is logarithmic. Combined with the large number of detected vortices in each flow case, the logarithmic binning allows for observation of six orders of magnitude in probability.

The estimated p.d.f.s of prograde vortex core diameter $p_{d}$ for each experimental dataset are shown in figure 5 . The p.d.f.s are shown with $d_{\omega}$ normalized by both the Kolmogorov length $\eta(a)$ and the Taylor microscale $\lambda_{T}(c)$. Figure 5 includes vortex cores from the entire outer layer, including the logarithmic and wake regions. Prior to constructing the histograms, the fitted vortex diameters were individually normalized using the average parameters $\eta\left(z_{\omega}\right)$ and $\lambda_{T}\left(z_{\omega}\right)$ local to the vortex wall-normal position $z_{\omega}$. Later figures presenting vortex core statistics also feature contributions from throughout the outer layer and utilize the individual normalization described here. Note that limiting the statistics to vortex cores at a specific wall-normal position does not affect the figure trends or the conclusions of the study.

Previous studies have described the most probable vortex size, i.e. the distribution mode, in terms of $\eta$. The mode for the DNS case in figure 5(a) is approximately $10 \eta$, which is between the values (8-10) $\eta$ observed by Tanahashi et al. (2004) and (12-13) $\eta$ by Herpin et al. (2013). While the observed range (10-20) $\eta$ in the mode across the remaining cases in figure 5(a) may be physically meaningful, it may also be attributed to variability in measurement resolution. Appendix A demonstrates that coarsening the DNS grid to $\Delta x^{+}$ values similar to the experimental cases results in the same observed range in mode position. The cross-hatched lines in figure 5 illustrate the diameter range where a strict comparison across cases is precluded by the influence of measurement resolution on the 

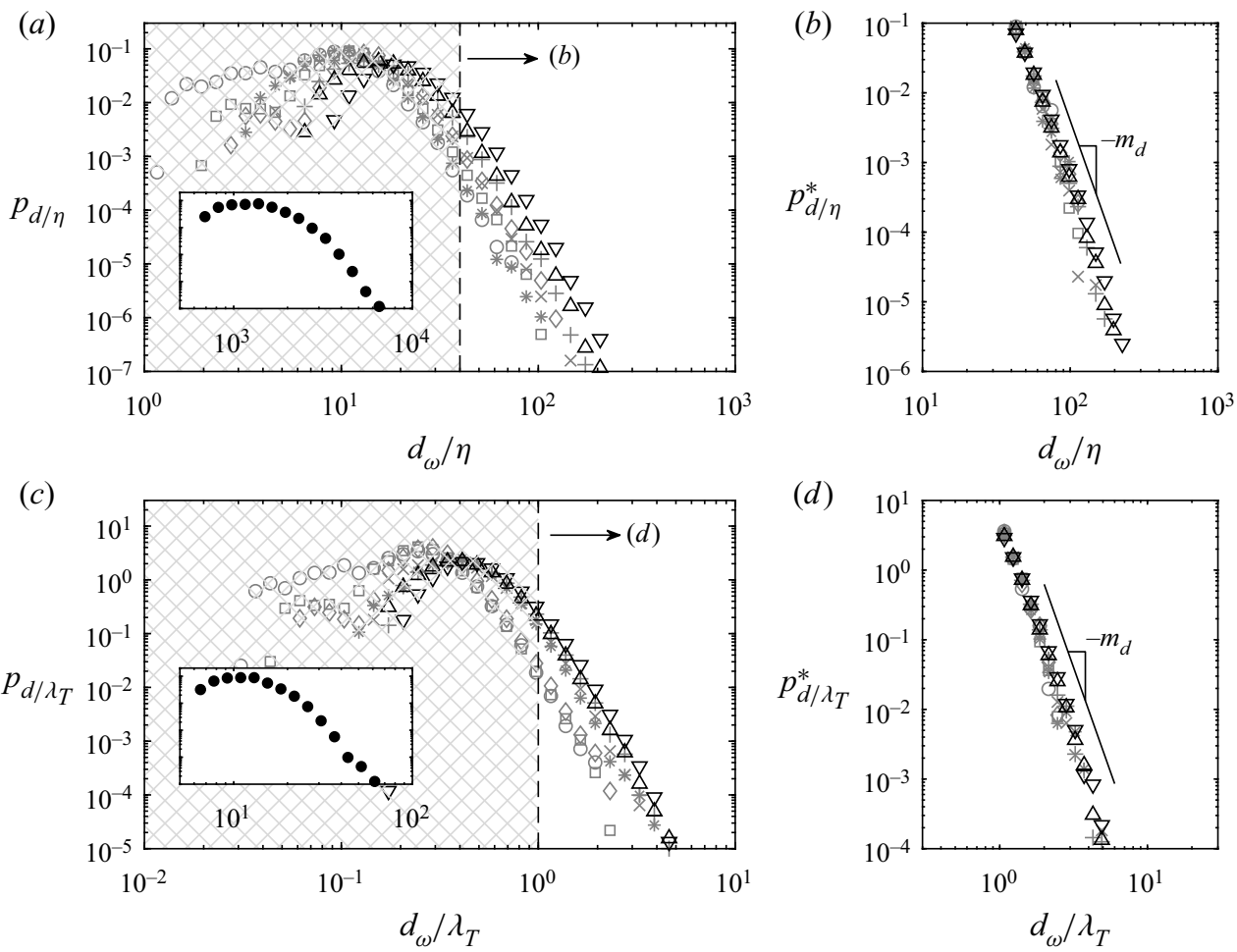

Figure 5. Probability distributions $p_{d}$ of diameter for all prograde vortex cores in the outer layer of the boundary layer: (a) p.d.f. normalized by the Kolmogorov length scale $\eta$; $(b)$ conditional p.d.f. $p_{d / \eta}^{*}=p_{d / \eta}\left(d_{\omega} \mid\right.$ $\left.d_{\omega}>40 \eta\right) ;(c)$ p.d.f. normalized by the Taylor microscale $\lambda_{T} ;(d)$ conditional p.d.f. $p_{d / \lambda_{T}}^{*}=p_{d / \lambda_{T}}\left(d_{\omega} \mid d_{\omega}>\right.$ $\left.\lambda_{T}\right)$. Comparison of results in the hatched regions of $(a, c)$ is precluded by variability in measurement resolution across cases, including the ASL case shown in the insets. The conditional p.d.f.s in $(b, d)$ are used to evaluate the distribution tail shape parameterized by the slope $m_{d}$. Data symbols correspond to the experimental datasets in table 1.

experimental cases with coarser $\Delta x$. Additionally, while there is an orders-of-magnitude difference in detected vortex size for the ASL case in the insets of figure $5(a, c)$, the difference is consistent with the orders-of-magnitude increase in $\Delta x$ seen in table 1 . We therefore draw no conclusions regarding either the distribution mode across cases or the detected size of ASL vortex cores.

Relative to the DNS case, some of the experimental results exhibit an increased number of vortices smaller than $10 \eta$ in figure 5(a). This increased detection of small vortices is likely an artefact of residual measurement noise. While it is possible to further quantify and filter the noise (see, e.g. Foucaut, Carlier \& Stanislas 2004), its effect is confined to the hatched region of figure 5 which is already subject to resolution bias and is excluded from further analysis.

Regarding the distribution shape, Herpin et al. (2013) showed that the size probability $p_{d}$ approximately follows a log-normal distribution, except with a somewhat thicker tail than the log-normal case. The figure 5 distribution tails are consistent with these findings. The tails deviate from a purely log-normal distribution and instead approximate a power law. The distribution tails shown in figure $5(b, d)$ are conditional p.d.f.s, e.g. $p_{d / \eta}^{*}=p_{d / \eta}\left(d_{\omega} \mid\right.$ $d_{\omega}>40 \eta$ ), which describe the probability given the diameter is above the threshold shown as a dashed line in figure $5(a, c)$. The conditional p.d.f. is equivalent to taking $p_{d}$ above the 


\section{Heisel, C.M. de Silva, N. Hutchins, I. Marusic and M. Guala}

threshold and rescaling to achieve area unity under the tail region. The rescaling removes the statistical contribution of the small-diameter vortices - which are affected by spatial resolution and measurement noise - to the amplitude of the normalized distribution tails. Given that the tail shape is not affected by resolution (see Appendix A), the $p_{d}^{*}$ plots allow for an unbiased comparison across cases for a consistent range of diameters.

The power law shape of the distribution tail is given by $p_{d}^{*} \propto d_{\omega}{ }^{-m_{d}}$. The collapse of the tails in figure $5(b, d)$ is due to a consistent slope $m_{d} \approx 6-7$ across cases, suggesting $m_{d}$ is independent of Reynolds number and the surface roughness geometries tested here. The slope is the same for the ASL case, and also does not vary with wall-normal position. The steepness of the slope $m_{d} \approx 6-7$ imposes an effective maximum value on the vortex core size, where the probability density decreases by orders of magnitude and becomes negligibly small across a narrow range of sizes. However, the relevant scaling parameter cannot be determined by the shape of the power law, as $m_{d}$ is independent of the normalization as seen in figure $5(b, d)$. In this regard, the diameter of the largest detected vortex cores may be $O(100 \eta)$ as in figure $5(b)$ or $O\left(1-10 \lambda_{T}\right)$ as in $5(d)$.

To discern the scaling parameter relevant to the largest vortex cores, the curvature of $p_{d}$ in the region preceding the power law must be considered. Given the sensitivity of the small-diameter vortex statistics to the measurement resolution (Appendix A), it is not possible to separate a physical trend in the figure $5 p_{d}$ curvature from the variability of resolution across the experiments studied here. These experimental factors can be avoided by focusing the analysis on the DNS flow case. Owing to the Reynolds dependence $\eta / \lambda_{T} \sim$ $\operatorname{Re}_{L}^{-1 / 4}$ where $\operatorname{Re}_{L}=u^{\prime} L / v$, the $d_{\omega}$ statistics for the DNS case were grouped in intervals of $R e_{L}$ based on the local mean values for $u^{\prime}$ and $L$ at the vortex position $z_{\omega}$.

Figure $6(a, b)$ shows distributions of $d_{\omega}$ for eight intervals of $R e_{L}$ within the DNS case. Having already demonstrated the power law shape of the probability tail in figure 5, the abscissa is shown with linear scaling in figure $6(a, b)$ to highlight amplitude differences along the tail. With $\eta$ normalization, there is a clear Reynolds number trend in the distribution tail region corresponding to large diameter vortex cores. Normalization by $\lambda_{T}$ in figure 6(b) leads to improved agreement across the range of $R e_{L}$ represented. There is no apparent trend in Reynolds number along the tails of $p_{d / \lambda_{T}}$ and the remaining narrow scatter is attributed to incomplete statistical convergence.

The general collapse of $p_{d / \lambda_{T}}$ in $6(b)$ suggests the largest vortex cores are confined by the same scaling that describes the ISL thickness. Considering also the steep slope $m_{d} \approx 6-7$ in figure 5 , the results indicate that the vortex core size is bounded between $\eta$ and $\lambda_{T}$ (Davidson 2015). Note that this conclusion applies only to strongly rotating prograde spanwise vortex cores. The strict detection criteria introduce selection biases which exclude larger, weakly rotating motions such as bulges and, as discussed later in $\S 5.3$, likely under-detects Kolmogorov-scaled vortices.

\subsection{Vortex inferred rate of strain}

Figure $6(b)$ provides promising results regarding the linkage between $\lambda_{T}$ and the size of the largest spanwise vortex cores. This relation is explored further here by assessing the rate of strain $\alpha$ acting on the detected vortices. The strain can be inferred using the radius $r_{\omega}$ fitted previously by the Oseen model along with the Burgers vortex radius definition $r_{\omega}=$ $\sqrt{4 v / \alpha}$ given in $\S 2.3$. The vortices are described here in terms of a strain rate $\alpha$ rather than a time $t$ (as in the Oseen model) to emphasize the physical relevance of the strain field. In this sense, the present section provides insight into the straining motions corresponding to the largest vortex cores and the vortex size that results from these motions. 

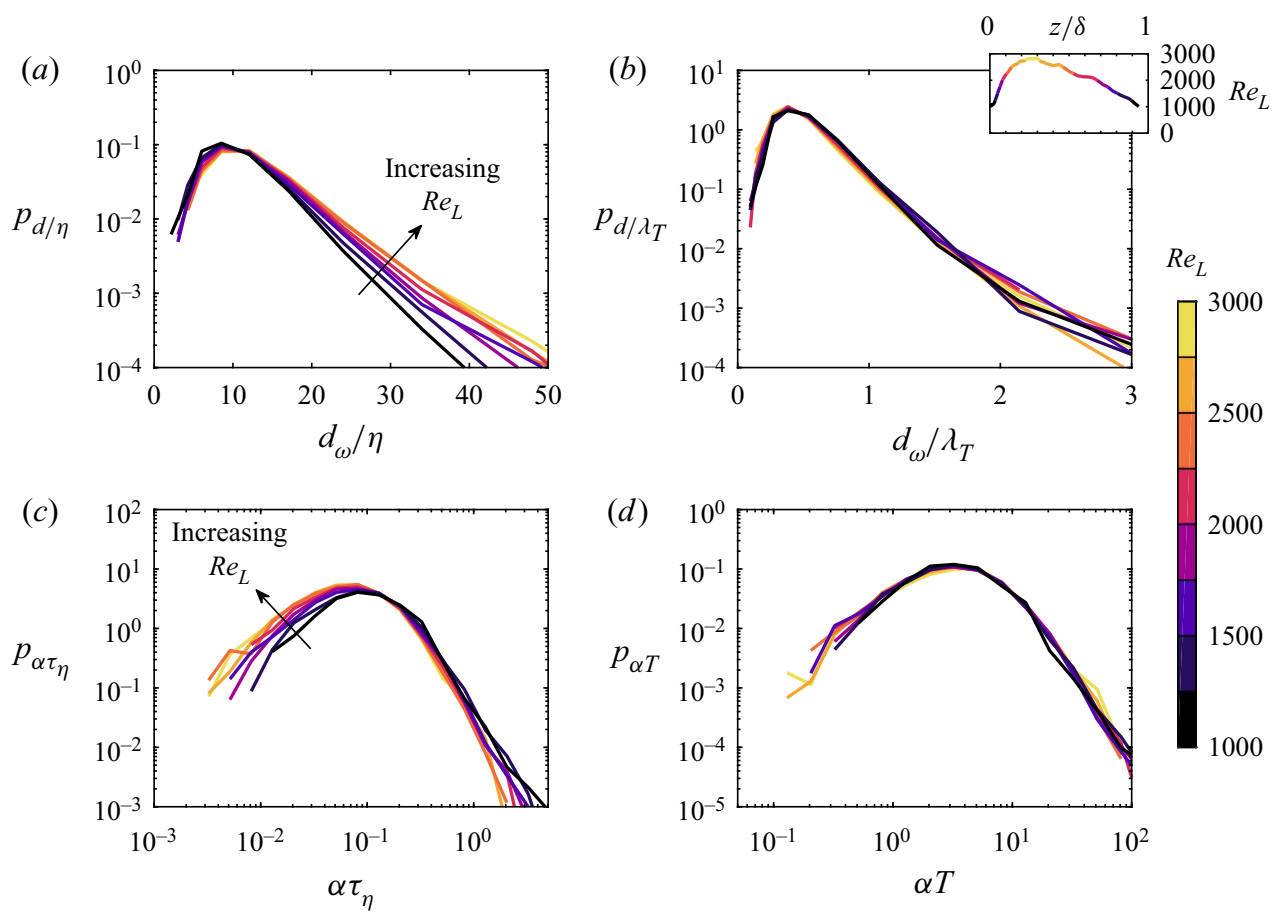

Figure 6. Probability distributions of the vortex core diameter $d_{\omega}$ and strain rate $\alpha=16 v d_{\omega}{ }^{-2}$ inferred from the Burgers vortex. Diameter p.d.f. $p_{d}$ normalized by $(a)$ the Kolmogorov length scale $\eta$ and $(b)$ the Taylor microscale $\lambda_{T}$. Strain rate p.d.f. $p_{\alpha}$ normalized by $(c)$ the Kolmogorov time scale $\tau_{\eta}$ and $(d)$ the large-eddy turnover time $T$. Data shown are for the DNS case in intervals of the integral Reynolds number $R_{L}$ determined by the local flow properties at the wall-normal position of the vortex. The upper right plot shows a profile of $R e_{L}$ for reference.

The relevant parameters for a strain rate comparison are the Kolmogorov time scale $\tau_{\eta}=$ $\sqrt{v / \epsilon}$ and large-eddy turnover time scale $T=L / u^{\prime}$ introduced in $\S 2.2$. Inserting these time scales into the expression $d_{\omega} \sim \sqrt{v t}$ returns the Kolmogorov length scale $\sqrt{\nu \tau_{\eta}}=$ $\left(v^{3} / \epsilon\right)^{1 / 4}=\eta$ and Taylor microscale $\sqrt{\nu T}=L / \sqrt{R_{L}} \sim \lambda_{T}$, respectively. An important advantage of considering the strain rate is the amplified Reynolds dependence $\tau_{\eta} / T \sim$ $R e_{L}^{-1 / 2}$.

As with the diameter statistics, an objective comparison of strain rates across experimental cases is precluded by the variability in experimental factors. Instead, the size analysis of DNS vortices in figure $6(a, b)$ is repeated for the strain rate in $6(c, d)$. The strain rate was estimated by inverting the Burgers vortex definition as $\alpha=16 v d_{\omega}{ }^{-2}$. Statistics for $\alpha$ are presented using the same intervals of $R e_{L}$ as for $d_{\omega}$. By using the vortex wall-normal position $z_{\omega}$ to determine the relevant parameter values, it is assumed that the net effect of non-local eddies is captured in their contribution to the local values for $T$ and $R e_{L}$. In other words, the local parameters $T\left(z_{\omega}\right)$ and $\operatorname{Re}_{L}\left(z_{\omega}\right)$ are assumed to be representative of the average eddy inducing strain on the vortex.

The normalized strain rate distributions in figure $6(c, d)$ are consistent with the vortex diameters in $6(a, b)$. The Reynolds-number trend in figure $6(c)$ corresponds to vortices with low strain rate and large diameter. Normalization of the strain by $T$ in figure $6(d)$ accounts for the $R e_{L}$ dependence and yields reasonable collapse of the distributions. The normalization by $T$ also provides better agreement than the mean shear time scale 


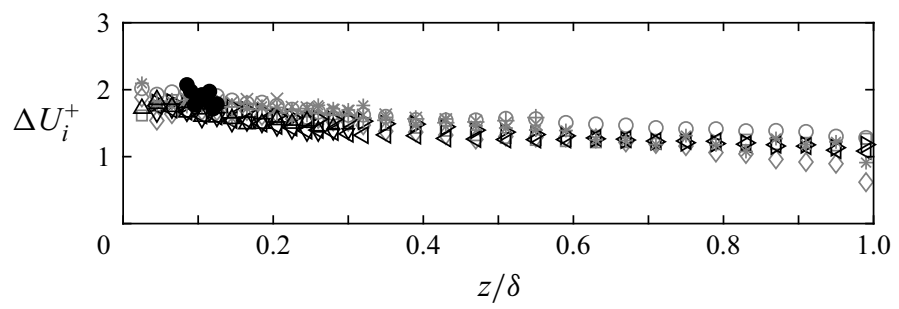

Figure 7. Wall-normal profile of the average streamwise velocity difference $\Delta U_{i}$ across ISLs. From figure 4 of Heisel et al. (2020b). Data symbols correspond to the experimental datasets in table 1.

$(\partial U / \partial z)^{-1}$ (not shown here) which overcompensates for the Reynolds-number trend seen in figure $6(c)$. Note that grouping the strain statistics in intervals of $z / \delta$ rather than $R e_{L}$ is less successful due to the non-monotonic profile of $\operatorname{Re}_{L}(z)$ shown in the upper right plot of figure 6 . The result suggests that the low-magnitude strain imposed on the detected Oseen-type vortices corresponds to a large-eddy time $T$ instead of Kolmogorov scales, and further supports $\lambda_{T}$ as the relevant parameter for describing the size of the largest vortex cores in the outer layer.

\section{Velocity statistics}

The primary velocity properties presented in the current section are the velocity differences: the streamwise velocity difference across the ISLs $\Delta U_{i}$ and the azimuthal velocity difference across the vortex diameter $\Delta u_{\omega}$. Statistics on the mean flow in the direction of the ISL and vortex centres are also provided. Results on the advection velocities of the detected structures are not given here, but are available elsewhere (Heisel 2020).

\subsection{Shear layer streamwise velocity}

The ISL average characteristic velocity $\Delta U_{i}$ is equivalent to the velocity difference between the UMZs bounding the shear layer. A wall-normal profile of this UMZ velocity, estimated using binned intervals of $z / \delta$, was previously given in figure 4 of Heisel et al. (2020b). The profile of $\Delta U_{i}$ is reproduced here as figure 7 for convenience. As noted in Heisel et al. (2020b), $\Delta U_{i}$ remains proportional to $u_{\tau}$ throughout the outer layer, including for the TNTI in the wake region. The velocity decreases moderately across the boundary layer. The scaling and wall-normal trend are both consistent with previous studies (de Silva et al. 2017; Gul et al. 2020). As a reminder, probability distributions of $\Delta U_{i}$ are not available due to the conditional averaging technique used to estimate the parameter.

\subsection{Vortex azimuthal velocity}

The velocity probability distributions for prograde vortex cores are shown in figure 8 using the same format as the diameter p.d.f.s. The distributions again contain several decades of probability which facilitates characterization of the shape. Unlike the size statistics, the velocity distributions are shown with linear spacing of the abscissa. The distribution tails are consistently linear in this plotted format, indicative of exponential tails given by $p_{u} \propto \exp \left(-\Delta u_{\omega} / m_{u}\right)$, where $m_{u}$ characterizes the linear slope of the tail seen in the figure. Variability in the $p_{u}$ mode is in part explained by differences in spatial resolution as demonstrated in Appendix A, most notably for the ASL case. Thus, no conclusions are 

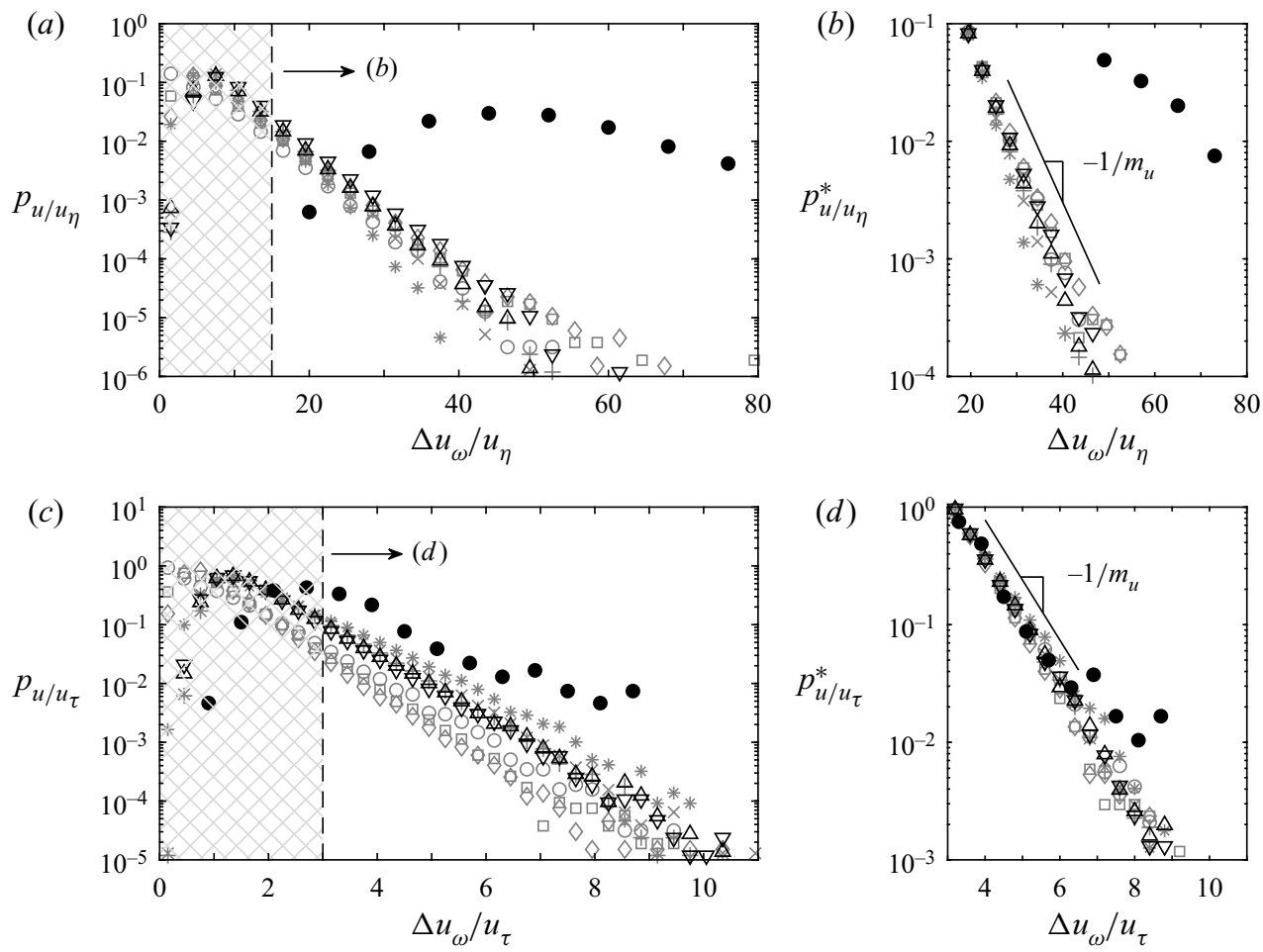

Figure 8. Probability distributions $p_{u}$ of azimuthal velocity for all prograde vortex cores: $(a)$ p.d.f. normalized by the Kolmogorov velocity $u_{\eta} ;(b)$ conditional p.d.f. $p_{u / u_{\eta}}^{*}=p_{u / u_{\eta}}\left(\Delta u_{\omega} \mid \Delta u_{\omega}>15 u_{\eta}\right)$; (c) p.d.f. normalized by the friction velocity $u_{\tau} ;(d)$ conditional p.d.f. $p_{u / u_{\tau}}^{*}=p_{u / u_{\tau}}\left(\Delta u_{\omega} \mid \Delta u_{\omega}>3 u_{\tau}\right)$. Comparison of results in the hatched regions of $(a, c)$ is precluded by variability in measurement resolution across cases. The conditional p.d.f.s in $(b, d)$ are used to evaluate the distribution tail shape parameterized by the slope $1 / m_{u}$. Data symbols correspond to the experimental datasets in table 1.

drawn here regarding the velocity mode, except to note the mode position may also be dependent on the vortex wall-normal position.

Same as for the vortex diameter statistics, figure $8(b, d)$ shows conditional p.d.f.s based on the thresholds given as dashed lines. The distribution tails given by $p_{u}^{*}$ indicate $u_{\tau}$ to yield somewhat better agreement in the tail slope than $u_{\eta}$ across cases, including for the ASL case. The tail slope is given as $1 / m_{u}$ rather than the reciprocal because the mean of an exponential distribution is defined by $m_{u}$. The probability $p_{u}$ is not fully exponential, as indicated by the presence of the mode, and $m_{u}$ therefore underestimates the mean in this case. However, $m_{u}$ is the relevant parameter for the long distribution tail and is likely important to the central moment statistics such as the average. Hereon, $m_{u}$ is referred to as a 'shape parameter' for the velocity distribution.

The value of $m_{u}$ was estimated for each flow case using a linear fit to the $p_{u}^{*}$ tails. The fits were specifically applied to the regions $\Delta u_{\omega}=(15-40) u_{\eta}$ and (3-6) $u_{\tau}$ where the probability statistics show better convergence. In addition to the p.d.f.s shown in figure 8 which include the vortices throughout the outer layer, $m_{u}$ was also estimated for velocity p.d.f.s in intervals of the wall-normal position based on the vortex centre $z_{\omega}$. The resulting $m_{u}$ values are shown in figure 9.

The shape parameter $m_{u}$ increases with Reynolds number $R e_{\tau}$ when normalized by $u_{\eta}$, and is approximately constant across three decades of $R e_{\tau}$ when normalized by $u_{\tau}$ 

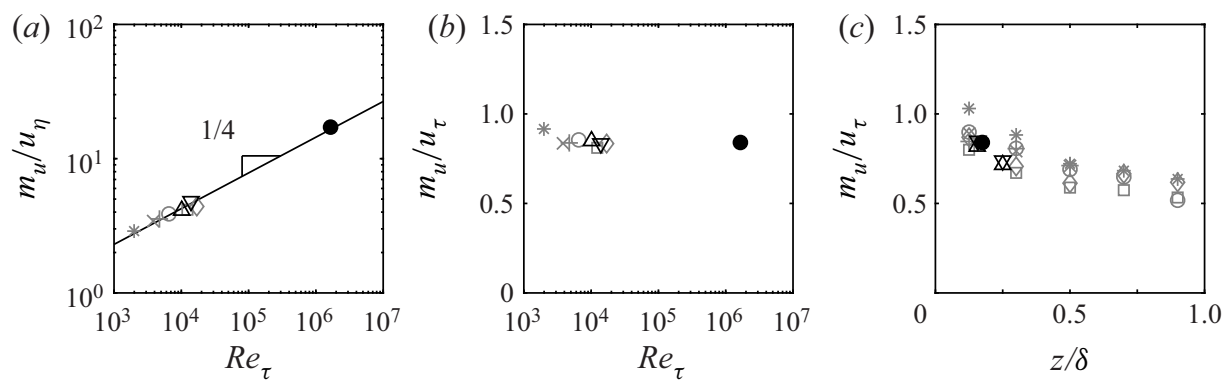

Figure 9. The shape parameter $m_{u}$ describing the exponential probability tail $p_{u} \propto \exp \left(-\Delta u_{\omega} / m_{u}\right)$ for the vortex azimuthal velocity: $(a, b)$ as a function of $R e_{\tau}$ when normalized by $u_{\eta}$ and $u_{\tau}$, respectively; $(c)$ wall-normal profile normalized by $u_{\tau}$. Data symbols correspond to the experimental datasets in table 1 .

in figure $9(b)$. This again supports the $u_{\tau}$ scaling for the probability tail suggested visually in figure 8. Similar to the scaling analysis for $d_{\omega}$, a weak Reynolds-number dependence is expected for the velocity scale separation. Given $u_{\tau}$ and $\delta$ are the same order as the respective integral scales $u^{\prime}$ and $L$ in the outer layer, the velocity scales are related as $u_{\tau} / u_{\eta} \sim R e_{\tau}^{1 / 4}$. Figure $9(a)$ supports this relationship, where the shape parameter exhibits the same weak Reynolds-number dependence $m_{u} / u_{\eta} \sim \operatorname{Re}_{\tau}^{1 / 4}$. The velocity tail statistics are better converged with less uncertainty across cases than the diameter mode position, and the $R e_{\tau}$ trend is apparent even within the laboratory datasets in figure $9(a)$.

In the wall-normal profile of $m_{u}$ in figure $9(c)$, the shape parameter decreases moderately with increasing $z$. The trend matches closely with $\Delta U_{i}(z / \delta)$ in figure 7 , where $m_{u} \approx$ $0.5 \Delta U_{i}$ throughout the outer layer. Given $m_{u}$ represents an underestimated value of the statistical mean $\Delta U_{\omega}$ for the modified exponential distributions in figure 8 , the azimuthal velocity of large vortices follows the same scaling and is quantitatively similar to the shear layer velocity $\Delta U_{i}$, indicating a close dynamical relationship between the largest prograde vortex cores and the ISLs.

The vortex statistics presented thus far, i.e. $d_{\omega}$ and $\Delta u_{\omega}$, are not independent from one another. The relation between the vortex core size and velocity is explored in Appendix B. The appendix results show that the population of detected vortex cores within the distribution tail of $p_{d}$ coincide with vortices also within the tail of $p_{u}$. The vortex cores exhibiting $u_{\tau}$ velocity scaling are therefore the same cores for which the influence of $\lambda_{T}$ is observed.

\subsection{Radial and wall-normal velocities}

We now turn the focus to the velocity components indicating flow to and from the vortex cores and shear layers in the $x-z$ measurement plane. These velocities, which are relative to the advection velocity of the considered flow regions, provide insight into the temporal evolution of these features in a more Lagrangian sense. In the case of the vortex cores, the relevant component is the radial velocity $u_{r}$ in cylindrical coordinates relative to the vortex centre. For each detected vortex core, the local flow field around the fitted vortex centre was compiled to create a profile of $u_{r}(r)$ as a function of radial distance $r$ from the vortex centre. The results were filtered to only include vortex cores larger than the mode for each dataset $\left(d_{\omega} \gtrsim 10 \eta \approx 0.3 \lambda_{T}\right)$ which are the focus of the study. A limited number of measurement points exist within the radius of the small vortices such that it is difficult to determine their radial trend. To average the profile across all the remaining 

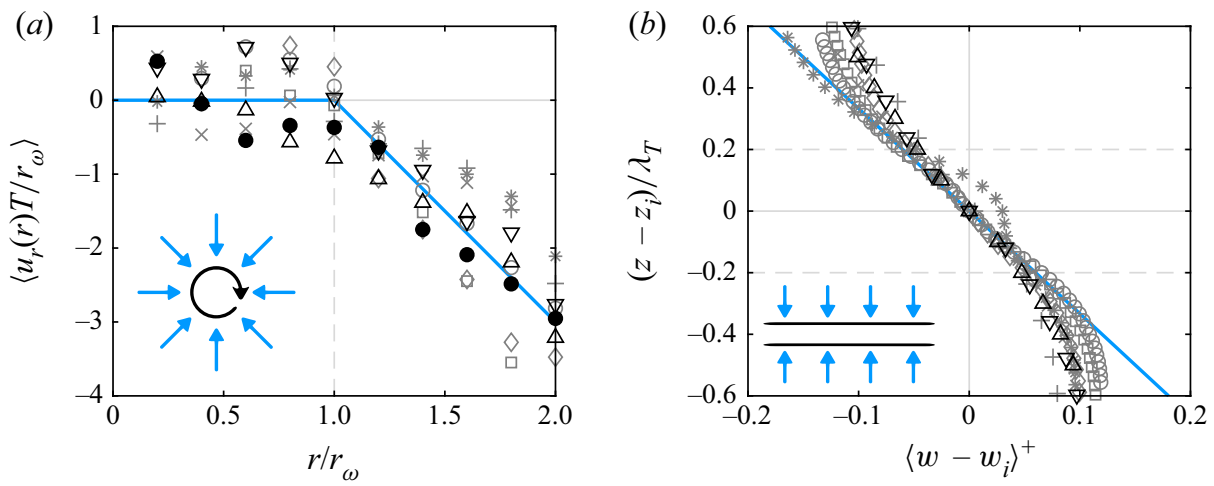

Figure 10. Average velocity profiles demonstrating the mean flow into the vortex cores and ISLs in the $x-z$ plane. (a) Vortex radial velocity $u_{r}(r)$ relative to the vortex centre $r=0$, normalized by its radius $r_{\omega}$ and the turnover time scale $T$. (b) Wall-normal velocity $w$ relative to the ISL velocity $w_{i}$ and midheight $z_{i}$, normalized by the Taylor microscale $\lambda_{T}$ and the friction velocity $u_{\tau}$. The dashed lines correspond to the average vortex radius $(a)$ and ISL thickness $(b)$. Data symbols correspond to the experimental datasets in table 1 .

vortices in a given flow case, the distance $r$ was normalized by the radius $r_{\omega}$ such that $r / r_{\omega}=1$ corresponds to the edge of each vortex core. The resulting average radial profiles of $u_{r}$ are provided in figure 10 $(a)$. Note that the Oseen model fitted to the vortices assumes $u_{r}(r)=0$, and any deviations from zero thus indicate physical behaviour that overcomes the selection bias of the imposed model.

The average $u_{r}(r)$ profile is close to zero within the vortex core $\left(r / r_{\omega}<1\right)$ and follows a trend of increasingly negative flow outside the core. There is generally good agreement across datasets, including the ASL case, with $u_{r}$ normalized by $r_{\omega} / T$. Normalizing both axes by $r_{\omega}$ ensures the negative slope outside the vortex is not a function of the detected vortex size in the plot. Even though the field measurements only detect the largest vortex cores and likely augment their size to some extent due to the spatial resolution, the statistics for $\Delta u_{\omega}$ and $u_{r}$ in the very-high-Reynolds-number ASL case are entirely consistent with the vortex velocity trends in the laboratory-scale flows.

Assuming $1 / T$ is representative of the strain rate $\alpha$ for the largest vortex cores as suggested by figure $6(d)$, the proportionality of $u_{r}$ with $1 / T$ outside the vortices in figure $10(a)$ is consistent with the profile $u_{r}(r)=-\alpha r$ predicted by the Burgers vortex. The result confirms the flow field around the vortex on average moves towards the vortex core at a speed proportional to the large-eddy strain rate $1 / T$. By continuity, this inward flow must be balanced by axial, out-of-plane motion as discussed further in $\S 5$. The overall shape of the radial profile and apparent transition point at $r=r_{\omega}$ may in part be due to the fitted Oseen model or spatial resolution, and confirmation with alternative methods is required.

For the shear layers, the wall-normal velocity component $w$ indicates flow towards the layers. The shear layers have an average orientation approximately 15 degrees above horizontal (Squire 2017), consistent with ramp-like structures, but in the present analysis the ISLs are considered horizontal for simplicity. The same conditionally averaged ISL profiles as in figure 4(a) were computed for $w$. The average profile of $w$ relative to the ISL midpoint is shown for each flow case in figure 10(b), excluding the ASL measurements whose resolution $\Delta x$ is coarser than the range of $z-z_{i}$ in the plot.

The best agreement across cases is achieved using the same normalization parameters as for the streamwise velocity case: $\lambda_{T}$ for the relative position $z-z_{i}$ and $u_{\tau}$ for the relative 


\section{Heisel, C.M. de Silva, N. Hutchins, I. Marusic and M. Guala}

velocity $w-w_{i}$. In the ISL reference frame, the average flow above the ISL is moving down into the shear layer $\left(w<w_{i}\right)$ and the flow below is moving up into the shear layer $\left(w>w_{i}\right)$. These same trends were observed in previous studies (Eisma et al. 2015; de Silva et al. 2017).

The normalization of the velocity $w-w_{i}$ by the strain parameter $\lambda_{T} / T$ yielded relatively less agreement across experimental datasets compared to $u_{\tau}$. There may be multiple processes resulting in the figure $10(b)$ profile such that a straining mechanism does not govern the scaling of the average velocity. For instance, the ISLs on average have a small net positive wall-normal velocity $W_{i} \sim 0.1 u_{\tau}$ (de Silva et al. 2017). The average relative velocity $\left\langle w-w_{i}\right\rangle$ may therefore be a combined result of the ISL advection $W_{i}$ associated with shear stress events in addition to the local compressive flow into the ISL. For most of the flow cases, the average value of the strain parameter $\lambda_{T} / T$ in the $\log$ region is approximately half the average value of $W_{i}$, and the ratio decreases for increasing Reynolds number.

A notable deviation in figure $10(b)$ is the centre-most region of the profile for the DNS case where the wall-normal velocity is uniform, i.e. see the ' $*$ ' markers near $\left(w-w_{i}\right)^{+} \approx 0.25$. The velocity profile is asymmetric such that there is a larger velocity difference above the centre than below, and the DNS profile centre was offset to match the bulk velocity difference outside the ISL. We attribute the DNS profile shape to a possible Kolmogorov-scaled centre region of the shear layers, which is consistent with predictions (Chini et al. 2017; Montemuro et al. 2020). A similar uniform centre is apparent for some of the experimental cases when the profiles in figure $10(b)$ are further conditioned to only consider ISLs in the wake region far from the wall where the local $\eta(z)$ is larger and the resolution $\Delta x / \eta$ is improved. The same wall-normal velocity profile shape has also been observed in previous ISL studies (Eisma et al. 2015). A more precise analysis of this centre-most region of the shear layers is not included here, as the focus of the study is the overall thickness of the layers and their relation to $\lambda_{T}$.

\section{Discussion}

The following discussion provides an interpretation of the experimental evidence presented thus far. The interpretation includes dynamics that cannot be fully validated with the current experimental observations in the $x-z$ measurement plane. We interpret the detected large prograde vortex cores as structures closely associated with the internal shear layers. While the present results do not spatially correlate the vortex cores and ISLs, previous works observed a strong alignment between these features (Klewicki \& Hirschi 2004; Heisel et al. 2018). Additionally, the instantaneous shear $\partial u / \partial z$ at the vortex centres is significantly higher than the local mean shear at the same position, indicating many of the vortex cores coincide with the intense shear regions. Specifically, the shear local to the vortices is (on average) 5-10 times larger than the mean shear in the logarithmic region for each flow case, and the factor increases in the wake region. We therefore expect that vortex cores aligned with the ISLs are at least partially responsible for the signature of $\lambda_{T}$ and $u_{\tau}$ scaling apparent from the distributions in figures 6 and 8 .

\subsection{Mutual interaction of turbulent structures}

One of the more revealing results to this point is the possible influence of the large-eddy turnover time $T$ on the properties of the biggest detected prograde vortex cores, see, e.g. figures $6(b)$ and $10(a)$. Coupled with the importance of $u_{\tau}$ to the vortex azimuthal velocity as seen in figure $9(b, c)$, the results indicate the local larger-scale motions play a critical 
(a)

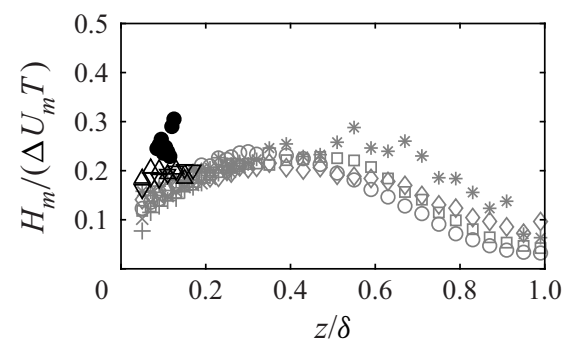

(b)

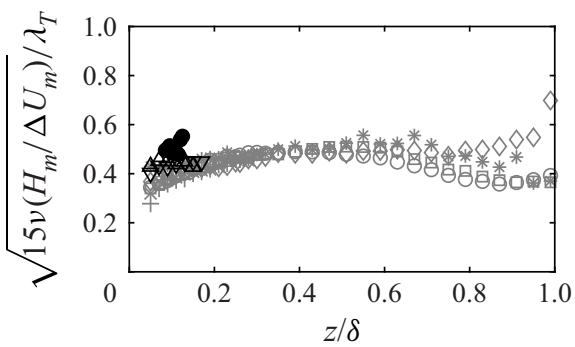

Figure 11. Wall-normal profiles of the turnover time estimated from average local UMZ properties $H_{m} / \Delta U_{m}$. (a) Compared with the large-eddy turnover time $T$. (b) Compared with the Taylor microscale $\lambda_{T}$ based on the isotropic relationship $\lambda_{T} \approx \sqrt{15 v T}$. Data symbols correspond to the experimental datasets in table 1 .

role in determining the properties of the largest vortex cores. However, the parameter $T$ was estimated from time-averaged flow properties and is not explicitly related to any type of coherent velocity structure such as UMZs.

To link $T$ with the local UMZs, we used the average UMZ profiles given in Heisel et al. (2020b) to construct a $z$-dependent 'UMZ turnover time' $H_{m}(z) / \Delta U_{m}(z)$, where $H_{m}$ is the average wall-normal thickness of detected UMZs, and $\Delta U_{m}=\Delta U_{i}$ is the average velocity difference across the shear layer between adjacent UMZs. The resulting profile is shown in figure 11. There is a consistent relationship between the UMZ turnover time and $T$ in the lower half of the outer layer in figure 11 $(a)$, with reasonable agreement across flow cases including the atmospheric measurements. While the proportionality changes moderately throughout the boundary layer, i.e. the normalized profile is not constant, the result suggests the contribution of the local momentum zones to the average parameter $T$ is statistically important. It is thus reasonable to assume the $T$-scaled strain field acting on the shear layers and vortex cores corresponds in part with the local UMZs. Note that previous studies found the lifetime of shear stress events in the log region to have the same scaling $z / u_{\tau}$ in the log region as the UMZ turnover time (Lozano-Durán \& Jiménez 2014). The trend in the wake region (and specifically $z \gtrsim 0.4 \delta$ ) can be explained by the influence of the free-stream condition on the statistics. The UMZ turnover time only considers structures detected below the TNTI, whereas $T$ is calculated using the full velocity series which in the wake region is a combination of boundary layer turbulence and the free stream.

The relation between $\lambda_{T}$ and $T$ is given by $\lambda_{T}=\sqrt{15 v T}$, where the constant 15 holds for isotropic turbulence as noted in $\$ 2.2$. Replacing $T$ with the UMZ turnover time in this definition, figure 11(b) compares the Taylor microscale estimated from local UMZ properties with the observed value for $\lambda_{T}$. The profile is relatively flat near the value 0.4 to 0.5 throughout the outer layer and there is again good agreement across flow conditions. Having already linked $T$ to the local UMZs, the result in figure 11(b) indicates a further connection between these UMZs and the size of the shear layers and large vortex cores along the UMZ interfaces.

The observed organization of the UMZs, shear layers and vortex cores in high-Reynolds-number turbulence is summarized in the figure 12(a) idealized profile. The UMZs have average thickness $H_{m}$ which is proportional to $z$ in the log region and transitions to outer $\delta$-scaling in the wake region (Heisel et al. 2020b). The average difference in streamwise velocity $\Delta U_{m}$ between the UMZs is proportional to $u_{\tau}$ throughout the outer layer, and decreases moderately with increasing $z / \delta$ (de Silva et al. 2017; Gul et al. 2020; Heisel et al. 2020b). The position of the largest spanwise vortex cores 


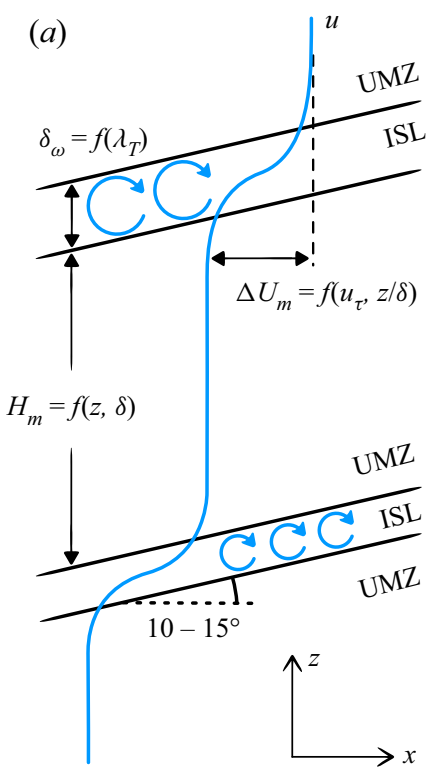

(b)

(1) Prograde vortex cores

(2) Streamwise rolls

(3) Stretching

(4) Viscous diffusion $\delta_{\omega} \sim \sqrt{v / \alpha}$

(5) Inward flow $\sim \alpha z$

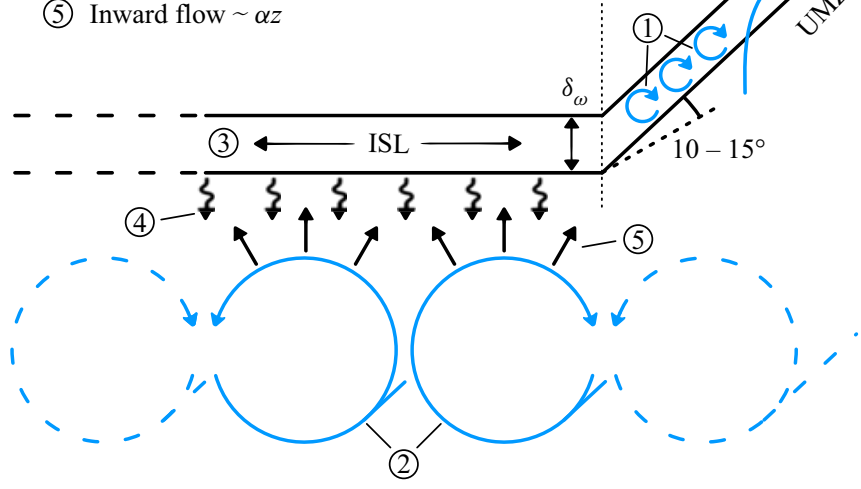

Figure 12. Simplified depiction of one possible mechanism for the interaction of coherent structures. $(a)$ The observed functional dependencies of the flow organization into relatively uniform velocity structures separated by inclined ISLs with corresponding spanwise prograde vortices. (b) Proposed interaction of velocity structures and vortices by the stretching mechanism, where the spanwise strain corresponds to counter-rotating streamwise rolls. The shear layer is depicted as a sheet with no variability in $y$ due only to lack of measurements in this plane. Dashed line structures represent the possible periodicity.

statistically coincides with the shear layers (Heisel et al. 2018). Both the shear layers and their corresponding large vortices have characteristic size $\lambda_{T}$ (figures 4 and 6) and velocity $u_{\tau}$ (figures 7 and 8). The evidence here suggests their size (figure 6), in addition to the radial velocity $u_{r}$ in the case of the vortices (figure 10), is closely related to properties of the local UMZs (figure 11).

The Burgers (1948) vortex model, already discussed multiple times in this work, can be invoked once again to formalize the interaction between the vortices, shear layers and UMZs. A key assumption in applying the full model here is the extension of the findings into the spanwise direction $y$. While the UMZ and large-eddy turnover times in figure 11 are based on flow properties measured in the $x-z$ plane, the Burgers model for spanwise vortices requires the UMZs to have a diverging spanwise velocity component to exert a tensile stress on the vortices. This diverging spanwise flow has been previously observed and associated with counter-rotating streamwise rolls. Studies have shown the roll signature to coincide with velocity structures - specifically side-by-side low- and high-velocity streaks - and share the same scaling behaviour in the log region as the UMZs (del Álamo et al. 2006; Lozano-Durán et al. 2012; Jiménez 2018). The velocity structures and their streamwise roll signature are depicted in relation to the shear layers and vortex cores in figure 12(b). Similar depictions of stretching are given in turbulence texts (e.g. Davidson 2015). Owing to the lack of spanwise measurements, the depiction shows no $y$ variation in the geometry of the shear layers and vortex cores. The three-dimensional shape of the interaction is a topic for future research.

To extend the findings into the spanwise plane as shown in figure $12(b)$, it is assumed that the streamwise turnover time $T$ is proportional to its spanwise counterpart. The strain field associated with the diverging flow of the streamwise rolls, with assumed scaling 
$\alpha \sim 1 / T \sim \Delta U_{m} / H_{m}$, then corresponds to spanwise stretching of the shear layers and large vortex tubes. The stretching induces flow into these features by continuity, where the velocity is proportional to $\alpha$, which is consistent with the findings in figure 10 . An approximate equilibrium is reached between the inward flow and the growth of the layers by viscous diffusion when the size is $\delta_{\omega} \sim \sqrt{v T} \sim \lambda_{T}$. The presence of diverging streamwise rolls beneath the ISLs is supported by recent experiments in the spanwise-wall-normal plane (Gul et al. 2020; Chen, Chung \& Wan 2021), and a similar mutual interaction of streamwise rolls and internal shear layers has been proposed based on asymptotic analysis in the limit of infinite Reynolds number (Chini et al. 2017; Montemuro et al. 2020).

The mechanism provides one possible pathway for the transfer of energy from larger-scale velocity structures into the dissipative shearing and vortical features. It also explains the observed size behaviour of the shear layers and large vortex cores. The equilibrium state of the size - as opposed to uninhibited diffusive growth as in the Oseen model - is consistent with the steep probability tail in $p_{d}\left(d_{\omega}\right)$ suggesting a confinement of the vortex size. Simple stretching models such as the Burgers vortex quickly reduce the size of large vortex tubes with $d_{\omega} \gtrsim O\left(\lambda_{T}\right)$ such that the observed range of sizes is effectively between $\eta$ and $\lambda_{T}$ (Davidson 2015). These findings are also consistent with visualizations of velocity fields in high-Reynolds-number boundary layers in which the presence of sharp shear layers is persistent in every instantaneous flow realization.

The primary purpose of figure $12(b)$ is to visualize the proposed interaction between UMZs and shear layers. It is important to note the depicted interaction is an incomplete description of the structural organization in boundary layer turbulence, and is likely one of several mechanisms relevant to the so-called self-sustaining process. The strain and stretching interaction does not address either the initiation or breakdown of the UMZ structures involved in the organization. It also does not explicitly account for the ramp-like inclination of the shear layers, their fractal geometry, or their three-dimensional shape. Future works involving spanwise measurements are required to evaluate the three-dimensionality and validate the axial (spanwise) stretching interaction in high-Reynolds-number wall turbulence. Further, while the depiction in figure 12 shares commonalities with reduced-order models including the wall-distance scaling and spanwise vortex heads of the attached eddy model (Marusic \& Monty 2019), analysis of three-dimensional experimental data is required to properly reconcile the present findings with these models.

Lastly, while only the diverging spanwise flow is discussed above, the counter-rotating streamwise rolls presumably also include converging flow regions (depicted by a dashed line in figure $12 b$ ) which would lead to vortex compression rather than stretching. Vorticity primarily aligns with the stretching and intermediate strain eigenvectors, and tends to tilt under compressive events (Guala et al. 2005; Holzner et al. 2010). If the vortex orientation is tilted by the converging flow of the rolls, the tilted vortices and the signature of their statistics would not be fully captured in the $x-z$ measurement plane. This may explain why stretching is statistically more relevant in the present results. The explanation assumes the phenomenology of small-scale strain vorticity interaction is not qualitatively different between isotropic and wall turbulence (see, e.g. Chacin \& Cantwell (2000), Tsinober (2001) and references therein).

\subsection{The relevance of the Taylor microscale in boundary layer turbulence}

For numerous decades, the Taylor microscale has been used as a statistical turbulence parameter, despite the elusiveness of its physical representation in the flow. Taylor (1935) 


\section{Heisel, C.M. de Silva, N. Hutchins, I. Marusic and M. Guala}

originally described the parameter as 'the average size of the smallest eddies responsible for the dissipation of energy'. While it has long been known this description is incorrect, as the smallest eddies correspond to the Kolmogorov scales, there has not been a widely accepted replacement to this phenomenological definition.

In the present findings, the intermediate length scale $\lambda_{T} \sim \sqrt{\nu T}$ emerges from the role of large-scale straining motions in bounding the size of the shear layers (figure 4) and largest vortex cores (figure 6). This process is depicted structurally in figure $12(b)$. The observations and the scaling definition above suggest the Taylor microscale statistically describes the size of turbulent features that are affected by both viscosity and the large-scale strain field. These features - the shear layers and large vortex cores - are in part governed by their mutual interaction with larger-scale coherent velocity structures such as UMZs. The role of viscosity in this interpretation is consistent with the definition for $\lambda_{T}$ given by da Silva et al. (2014). In addition to boundary layer ISLs (Eisma et al. 2015; de Silva et al. 2017), $\lambda_{T}$ scaling applies to the thickness of shear layers in isotropic turbulence (Ishihara et al. 2009; Elsinga et al. 2017), the TNTI in jet turbulence (da Silva \& Taveira 2010) and the boundary layer TNTI, as previously noted (Chauhan et al. 2014b).

By this interpretation, $\lambda_{T}$ is a dynamically important parameter related to coherent features in the flow, especially in high-Reynolds-number turbulence where these features are increasingly distinct from the Kolmogorov- and integral-scaled motions. This work contributes to the growing body of evidence presenting $\lambda_{T}$ as a relevant length scale. However, to confirm the role of intermediate scales like $\lambda_{T}$ in the phenomenology of turbulence, corroboration is required using high-resolution measurements and simulations across an increasing range in both Reynolds number and analysis methods.

\subsection{Vortex selection bias}

While the evidence here demonstrates the dynamics pertaining to the largest vortex cores, no arguments can be made regarding smaller vortices. There is a preponderance of worm-like, Kolmogorov-scaled vortices in isotropic turbulence (see, e.g. She et al. 1990; Jiménez et al. 1993; Moisy \& Jiménez 2004), and it is reasonable to expect similar features in boundary layer flows. However, there are a number of factors in the present study that reduce the detection of these small-diameter and low-velocity vortices in the respective $p_{d}$ and $p_{u}$ distributions. Foremost is the measurement spatial resolution whose influence is demonstrated in Appendix A.

Additionally, the detection methodology detailed in $\S 2.3$ directly imposes a selection bias, where the vortex statistics specifically reflect flow features that resemble the Oseen model vortex. Visual inspection of the fitted results revealed that many of the largest detected vortices comprise a cluster of high-intensity $\lambda_{c i}$ regions that together resemble a large Oseen vortex. For example, the core region in figure 2(a) may be a possible cluster of smaller vortices rather than a single concentric motion. Three-dimensional measurements and a different methodology such as the q-criterion based on the second invariant of the deformation tensor (Hunt, Wray \& Moin 1988) may lead to detection of the individual smaller vortices within the cluster. For instance, da Silva \& Taveira (2010) and da Silva et al. (2014) used two detection methodologies to identify separate populations of Kolmogorov-scaled vortex 'worms' within larger vortices whose size corresponded to the thickness of interfacial layers.

Finally, an indirect form of selection bias is orientation. Owing to the mean shear direction, the large vortex cores spanning the shear layers preferentially have spanwise out-of-plane rotation. In contrast, the smallest vortices may have less or no 


\section{Vortices and shear layers in turbulent boundary layers}

preferential direction. The detection of spanwise vortices in the $x-z$ plane therefore will identify many of the large vortex cores, but only a small portion of the small-scale vortices may appear in the given measurement plane. Based on the resolution limitations and selection biases, we restrict our conclusions to the detected large vortex cores, and note that the smallest vortices are under-detected in this study.

\section{Summary}

The properties of prograde spanwise vortex cores and ISLs in turbulent boundary layers are evaluated here across a range of experimental datasets including smooth- and rough-wall conditions and ASL measurements. The analysis corroborates previous studies on the scaling behaviour of both UMZ interfaces and internal shear layers (Eisma et al. 2015; de Silva et al. 2017; Gul et al. 2020), and confirms the scaling also applies for rough-wall flows. Probability distributions of the vortex core properties reveal signatures of these same scaling parameters on the vortex statistics. The largest detected vortex cores are confined in size by the Taylor microscale $\lambda_{T}$ (figure 6) and have azimuthal velocity governed by the friction velocity $u_{\tau}$ (figures 8 and 9). Selection biases inherent to the methodology preclude any conclusions regarding the smallest vortices, which are expected to be Kolmogorov scaled. The sensitivity of the vortex size statistics (figure 5) to experimental factors such as measurement resolution, which is especially penalizing for the field experiment, does not allow for an unambiguous scaling assessment of vortex core size across cases. Accordingly, quantitative support for the influence of $\lambda_{T}$ on the largest vortex cores is primarily provided by DNS results (figure 6). However, the $u_{\tau}$ velocity scaling of the large vortex cores is confirmed across all experiments, including the atmospheric measurements. The results demonstrate that ISLs and large prograde vortex cores throughout the entire outer layer are dependent on the wall shear condition defined by $u_{\tau}$.

Further analysis of the vortex properties suggests a dynamic connection between the largest vortex cores and local large-scale coherent velocity structures such as UMZs. The inferred strain rate acting on the largest vortex cores (figure 6) and the mean radial flow into these vortices (figure 10) both correspond to a large-eddy turnover time scale $T=u^{\prime 2} / \epsilon$. The relevance of an intermediate scale such as $\lambda_{T}$ can then be explained in terms of the simplified Burgers vortex model, where the size of the ISLs and largest vortex cores is determined by both viscous diffusion and the strain of local large-scale motions. The spanwise strain required to satisfy this model cannot be directly detected from the measurements presented here, but is assumed to be associated with pairs of counter-rotating streamwise rolls (figure 12).

The observed signature of the large-scale strain on smaller-scale structures also provides insight into the persistence of the self-organized UMZs and ISLs in high-Reynolds-number boundary layers. The ISLs and spanwise vortex cores are constrained such that a limited range of ISL thickness and vortex diameter are observed in every flow realization. This proposed interaction between the local larger-scale velocity structures, ISLs, and vortex cores features two key processes in turbulence evolution: vorticity production occurs via the vortex stretching mechanism, and energy production results from the coupling of the velocity with the large gradients across the shear layers. These interactions are inferred from the present observations in the streamwise-wall-normal measurement plane, and the three-dimensionality of the interaction should be investigated through further research.

Acknowledgements. The authors are grateful to J.A. Sillero and co-authors for allowing public access to the DNS data. 


\title{
M. Heisel, C.M. de Silva, N. Hutchins, I. Marusic and M. Guala
}

Funding. The authors acknowledge funding support from the Institute on the Environment (IonE) and the Australian Research Council. M.H. is supported by fellowships from the University of Minnesota Graduate School and the National Science Foundation (NSF-AGS-2031312). M.G. is supported by a National Science Foundation CAREER grant (NSF-CBET-1351303).

Declaration of interests. The authors report no conflict of interest.

\author{
Author ORCIDs. \\ (D) Michael Heisel https://orcid.org/0000-0002-4200-5550; \\ D Charitha M. de Silva https://orcid.org/0000-0001-9517-4318; \\ Nicholas Hutchins https://orcid.org/0000-0003-1599-002X; \\ Ivan Marusic https://orcid.org/0000-0003-2700-8435; \\ Michele Guala https://orcid.org/0000-0002-9788-8119.
}

\section{Appendix A. Spatial resolution effects on vortex probability statistics}

Given the smallest turbulent motions have size $O(\eta)$, the present analysis ideally would have used experiments and simulations at matched spatial resolutions with small $\Delta x / \eta$ or $\Delta x^{+}$. However, such a matched comparison was not feasible for the range of Reynolds number tested. We therefore present each case with its native resolution, and accept that variability in spatial resolution across the cases will influence certain statistics. The effect of resolution on vortex probability statistics is evaluated here to determine which results are (in)dependent of resolution and to confirm the validity of conclusions drawn in the main article.

The resolution test involved filtering the DNS velocity fields and reapplying the vortex detection on a coarsened grid. A box filter, equivalent to a two-dimensional moving average, was applied to achieve an effect similar to the averaging across a PIV interrogation window. The filtered field was then downsampled to achieve a grid spacing equal to the filter size. Figure 13 compares vortex statistics for the original DNS velocity fields and for three filter sizes which span the range of resolutions (in $\Delta x^{+}$) for the PIV experiments in table 1 .

The number density, calculated as the number of vortex cores in each histogram bin divided by the bin width, is shown for the vortex diameter and velocity in figure 13( $a, c)$, respectively. The number density indicates the change in detected vortices of each size and velocity due to the coarsened resolution. The primary effect of resolution on the vortex diameter is to decrease the number of small vortex cores detected, which additionally leads to a shift in the probability mode to a larger diameter. We therefore do not make any conclusions regarding the value of the probability mode or the distribution shape for diameters smaller than the mode.

A second effect of coarse resolution is to increase the number of large-diameter vortex cores detected. The increase is likely caused by multiple factors, most notably: (i) the size is augmented by smoothing effects across the filter (i.e. PIV window) which increases the vortex footprint; (ii) the low-pass filter reduces the swirling strength in the field, which decreases the $\lambda_{r m s}$ threshold and leads to the new detection of large and weak vortices. These results indicate the diameters for the ASL case in figure 5 represent only the largest vortex cores in the atmosphere, and the fitted diameters of $O\left(10-100 \lambda_{T}\right)$ are likely overestimated.

Similar to the diameter, coarsening the resolution leads to the detection of fewer small-velocity vortices in figure 13(c) and a shift in vortex velocity mode. Statistics for the velocity mode are therefore also unreliable due to a dependence of resolution. Unlike the 

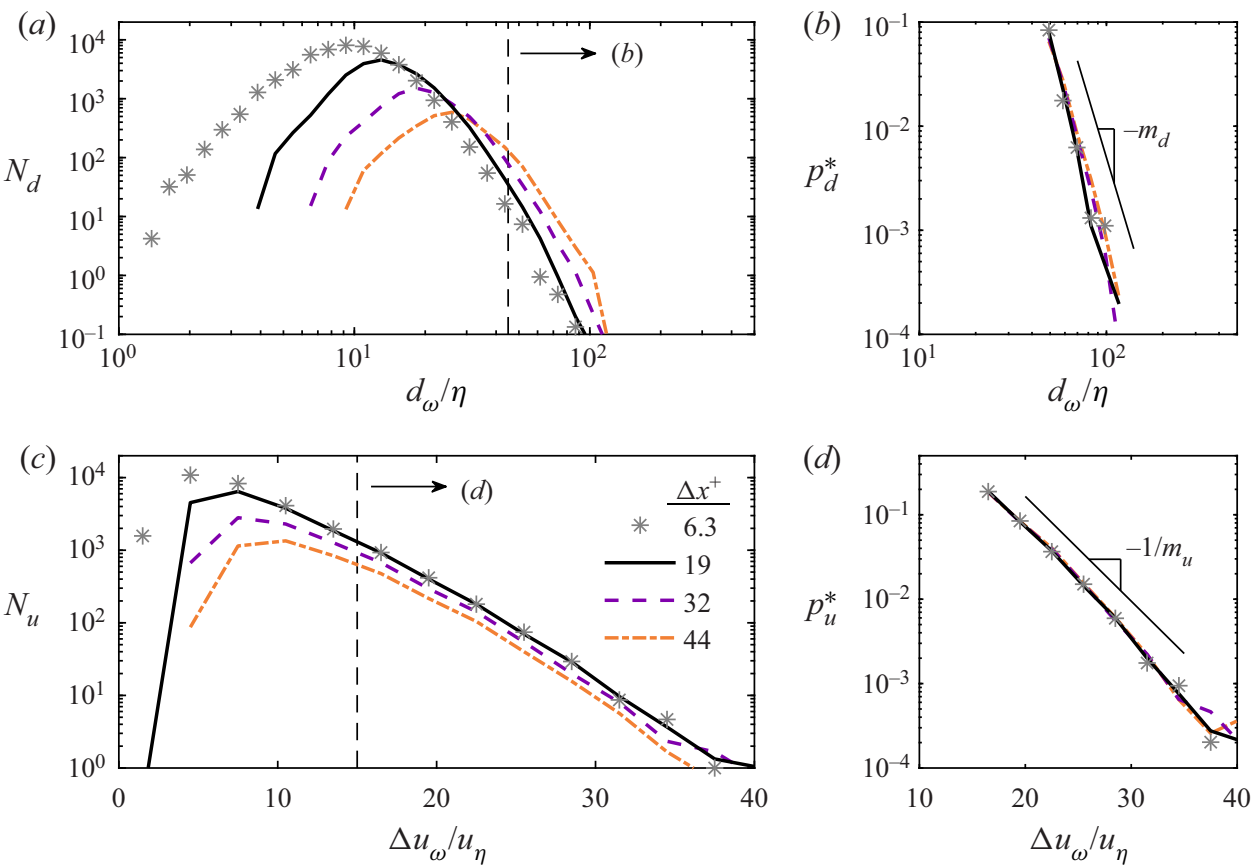

Figure 13. Probability distributions of vortex statistics for the DNS case filtered to coarsened grid resolutions: (a) number density $N_{d}$ of diameter; $(b)$ conditional p.d.f. $p_{d}^{*}$ of diameter probability tail; $(c)$ number density $N_{u}$ of velocity; $(d)$ conditional p.d.f. $p_{u}^{*}$ of velocity probability tail. Data markers are for the unfiltered DNS velocity fields, and lines indicate results from the DNS fields filtered and downsampled to the specified effective resolution.

diameter, however, the filter smoothing decreases the velocity difference across the vortex core and leads to a small decrease in number of detected large-velocity vortices.

Most importantly for the present study, the filter and spatial resolution do not change the shape of the probability tail. While the resolution influences the number of vortex cores with large diameter or azimuthal velocity, the tail slopes in the figure $13(b, d)$ conditional p.d.f.s are negligibly affected by the coarsened resolution. Conclusions of the study regarding the power law shape of the vortex diameter probability tails (figure 5) and the influence of $u_{\tau}$ on the velocity probability tails (figure 8 ) are thus considered to be independent of the variation in spatial resolutions across the cases.

\section{Appendix B. Vortex joint probability statistics}

In the Oseen model used to detect spanwise vortex cores, the vortex properties are related through the functional dependence $\Delta u_{\omega} \propto \Gamma d_{\omega}{ }^{-1}$ from Biot-Savart law. The circulation $\Gamma$ is also related to the total out-of-plane vorticity within the area of the vortex core: $\Gamma \propto$ $\omega_{y} d_{\omega}^{2}$. Combined with the first dependence, this yields an expected relationship $\Delta u_{\omega} \propto$ $\omega_{y} d_{\omega}$. The azimuthal velocity difference therefore increases with both the rate of rotation $\left(\omega_{y}\right)$ and size $\left(d_{\omega}\right)$.

The correlation of vortex size and velocity is apparent through the joint p.d.f. $p_{d, u}\left(d_{\omega}, \Delta u_{\omega}\right)$. An example joint p.d.f. for the $R e_{\tau}=3800$ smooth-wall case of table 1 is shown in figure $14(a)$. The shape of the individual p.d.f.s, including the position of their mode, are shown for reference. If the two variables are statistically independent 
(a)

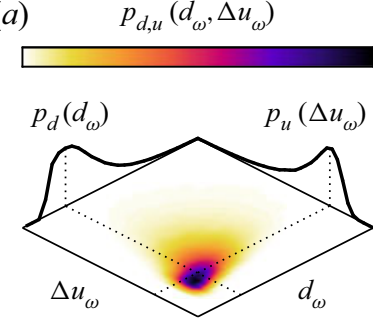

(b) $\quad p_{d}\left(d_{\omega}\right) p_{u}\left(\Delta u_{\omega}\right)$

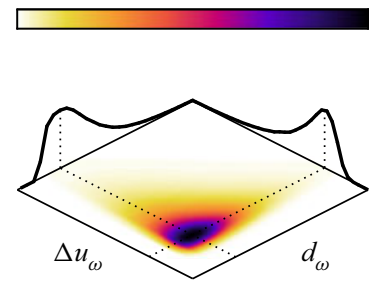

(c) $\tilde{p}_{d, u}\left(d_{\omega}, \Delta u_{\omega}\right)=p_{d, u} / p_{d} p_{u}$

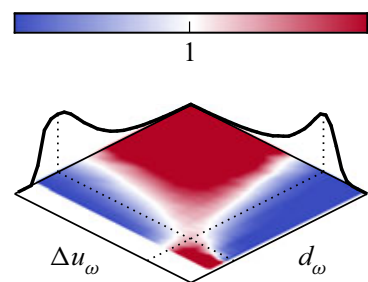

Figure 14. Example of the relative joint probability $\tilde{p}_{d, u}\left(d_{\omega}, \Delta u_{\omega}\right)$ of vortex size and velocity from the $R e_{\tau}=$ 3800 smooth-wall case. (a) Observed joint probability $p_{d, u}$ (colour) compared with the individual p.d.f.s (solid lines) and positions of the p.d.f. modes (dotted lines). (b) Product of the two individual p.d.f.s $p_{d} p_{u}$ (colour), representing the joint probability if $d_{\omega}$ and $\Delta u_{\omega}$ were statistically independent. (c) The relative probability $\tilde{p}_{d, u}$ (colour) from the ratio of the probabilities in $(a)$ and $(b)$.

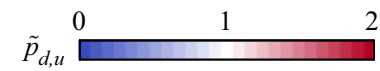

(a)

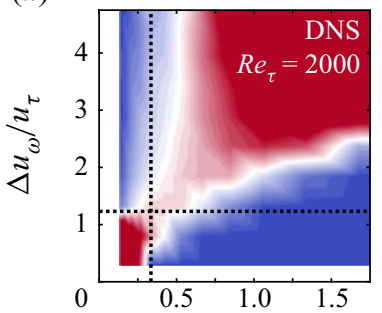

(b)

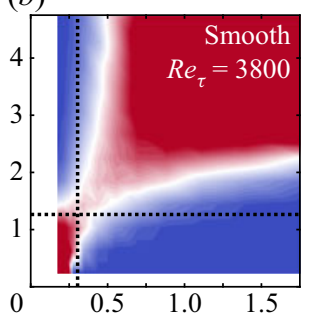

(c)

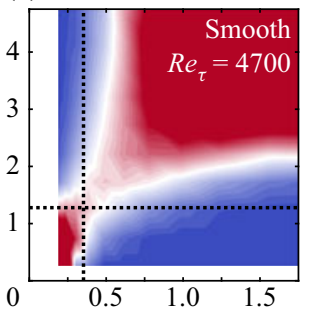

$(g)$

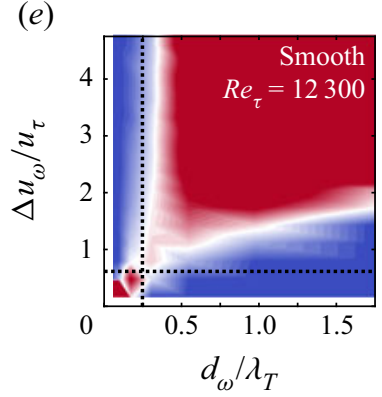

$(f)$

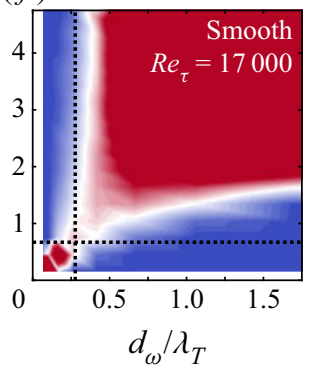

$(d)$

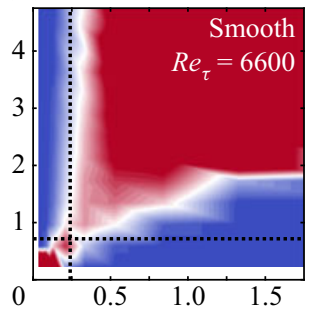

(h)

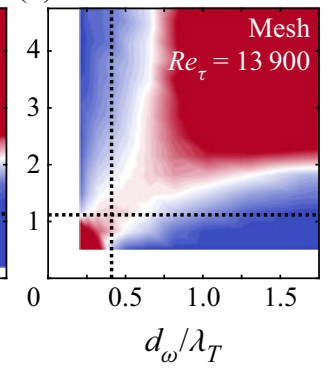

Figure 15. Following the example in figure 14 , relative joint probability $\tilde{p}_{d, u}\left(d_{\omega}, \Delta u_{\omega}\right)$ for each experimental case, excluding the ASL measurements. Dotted lines indicate the modes of the individual p.d.f.s. Each plot lists the wall condition and Reynolds number corresponding to table 1.

(i.e. uncorrelated), their joint p.d.f. is equal to the product of the individual p.d.f.s at every size and velocity: $p_{d}\left(d_{\omega}\right) p_{u}\left(\Delta u_{\omega}\right)$. This product is shown in the figure $14(b)$ joint distribution representing the independent case. There are clear visual differences between the colour plots in figure 14(a,b). The independent case in figure 14(b) is aligned with the individual p.d.f. modes, whereas the more linear shape of $p_{d, u}$ in $14(a)$ indicates the expected positive correlation.

A more quantitative analysis of the relationship is achieved by simply taking the ratio of the observed joint p.d.f. with the independent case

$$
\tilde{p}_{d, u}\left(d_{\omega}, \Delta u_{\omega}\right)=\frac{p_{d, u}\left(d_{\omega}, \Delta u_{\omega}\right)}{p_{d}\left(d_{\omega}\right) p_{u}\left(\Delta u_{\omega}\right)} .
$$




\section{Vortices and shear layers in turbulent boundary layers}

We refer to $\tilde{p}_{d, u}$ as the 'relative' joint probability, i.e. relative to the independent case, and note different terminology and notation may be used in the statistics literature. The $\tilde{p}_{d, u}$ values for the example case are shown in figure $14(c)$. For $\tilde{p}_{d, u}>1$ (red regions), the joint probability is more likely than if the variables were independent, and conversely the joint probability is less likely for $\tilde{p}_{d, u}<1$ (blue regions). More specifically, the value $\left(\tilde{p}_{d, u}-1\right) \times 100 \%$ provides the per cent change in probability from the uncorrelated case.

The relative joint probability is shown for each flow case in figure 15, excluding the ASL measurements. The figure confirms the positive correlation: larger vortex cores are more likely to have higher azimuthal velocity than in the independent case. The positive correlation is itself neither surprising nor particularly revealing. The more important result for the present analysis is the apparent presence of four distinct probability regions whose boundaries correspond approximately to the modes of the individual p.d.f.s. The region corresponding to the largest variability and uncertainty across experiments are the vortices smaller than the mode with $d_{\omega} \lesssim 0.3 \lambda_{T}\left(d_{\omega} \lesssim 10 \eta\right.$ at these Reynolds numbers), referred to as 'small-diameter' vortices in the present discussion. Figure 15 shows the probability of a small-diameter vortex with large velocity to be negligibly small, such that the population of detected small-diameter vortices also have $\Delta u_{\omega}$ values primarily confined below the mode. Conversely, the population of vortices corresponding to the probability tail of $p_{u}$ also have size within the large-diameter tail of $p_{d}$. The role of vorticity in determining $\Delta u_{\omega}$ does not decorrelate the $p_{d, u}\left(d_{\omega}, \Delta u_{\omega}\right)$ statistics to an extent where mixed magnitudes are commonly observed, e.g. a small-diameter vortex with large velocity. The probability tail trends for $p_{d}$ in $\S 3.2$ and $p_{u}$ in $\S 4.2$ therefore relate to the same population of detected vortex cores residing in the upper-right region of the figure 15 plots, where the evidence suggests the relevant scaling parameters are $\lambda_{T}$ (figure 6) and $u_{\tau}$ (figure 8).

\section{REFERENCES}

Adrian, R.J., Christensen, K.T. \& LiU, Z.-C. 2000a Analysis and interpretation of instantaneous turbulent velocity fields. Exp. Fluids 29 (3), 275-290.

Adrian, R.J., MeInhart, C.D. \& Tomkins, C.D. $2000 b$ Vortex organization in the outer region of the turbulent boundary layer. J. Fluid Mech. 422, 1-54.

Agostini, L. \& LeschZiner, M. 2017 Spectral analysis of near-wall turbulence in channel flow at $r e_{\tau}=$ 4200 with emphasis on the attached-eddy hypothesis. Phys. Rev. Fluids 2, 014603.

Del Álamo, J.C., Jiménez, J., Zandonade, P. \& Moser, R.D. 2006 Self-similar vortex clusters in the turbulent logarithmic region. J. Fluid Mech. 561, 329-358.

Bisset, D.K., HunT, J.C.R. \& Rogers, M.M. 2002 The turbulent/non-turbulent interface bounding a far-wake. J. Fluid Mech. 451, 383-410.

Brown, G.L. \& Roshко, A. 1974 On density effects and large structure in turbulent mixing layers. J. Fluid Mech. 64 (4), 775-816.

BuRgers, J.M. 1948 A mathematical model illustrating the theory of turbulence. In Advances in Applied Mechanics, vol. 1, pp. 171-199. Academic Press Inc.

CARlier, J. \& Stanislas, M. 2005 Experimental study of eddy structures in a turbulent boundary layer using particle image velocimetry. J. Fluid Mech. 535, 143-188.

Chacin, J.M. \& CAntwell, B.J. 2000 Dynamics of a low Reynolds number turbulent boundary layer. J. Fluid Mech. 404, 87-115.

Chakraborty, P., Balachandar, S. \& Adrian, R.J. 2005 On the relationships between local vortex identification schemes. J. Fluid Mech. 535, 189-214.

Chauhan, K., Philip, J. \& Marusic, I. 2014a Scaling of the turbulent/non-turbulent interface in boundary layers. J. Fluid Mech. 751, 298-328.

Chauhan, K., Philip, J., De Silva, C.M., Hutchins, N. \& Marusic, I. 2014b The turbulent/non-turbulent interface and entrainment in a boundary layer. J. Fluid Mech. 742, 119-151.

Chen, X., Chung, Y.M. \& WAn, M. 2021 The uniform-momentum zones and internal shear layers in turbulent pipe flows at Reynolds numbers up to $R e_{\tau}=1000$. Intl J. Heat Fluid Flow 90, 108817. 


\section{Heisel, C.M. de Silva, N. Hutchins, I. Marusic and M. Guala}

Chini, G.P., Montemuro, C., White, C.M. \& Klewicki, J.C. 2017 A self-sustaining process model of inertial layer dynamics in high Reynolds number turbulent wall flows. Phil. Trans. R. Soc. A 375 (2089), 20160090.

DAVIDSON, P.A. 2015 Turbulence: An Introduction for Scientists and Engineers, 2nd edn. Oxford University Press.

Ebner, R.L., Mehdi, F. \& KLEwiCKi, J.C. 2016 Shared dynamical features of smooth-and rough-wall boundary-layer turbulence. J. Fluid Mech. 792, 435-469.

EICH, F., DE Silva, C.M., MARUsiC, I. \& KÄHLER, C.J. 2020 Towards an improved spatial representation of a boundary layer from the attached eddy model. Phys. Rev. Fluids 5, 034601.

Eisma, J., Westerweel, J., OOMs, G. \& ElsingA, G.E. 2015 Interfaces and internal layers in a turbulent boundary layer. Phys. Fluids 27 (5), 055103.

Elsinga, G.E., Ishihara, T., Goudar, M.V., DA Silva, C.B. \& Hunt, J.C.R. 2017 The scaling of straining motions in homogeneous isotropic turbulence. J. Fluid Mech. 829, 31-64.

Elsinga, G.E. \& MARUSiC, I. 2010 Universal aspects of small-scale motions in turbulence. J. Fluid Mech. 662, 514-539.

ElsingA, G.E. \& DA Silva, C.B. 2019 How the turbulent/non-turbulent interface is different from internal turbulence. J. Fluid Mech. 866, 216-238.

FAN, D., XU, J., YAO, M.X. \& HiCKEY, J.P. 2019 On the detection of internal interfacial layers in turbulent flows. J. Fluid Mech. 872, 198-217.

Foucaut, J.M., Carlier, J. \& Stanislas, M. 2004 PIV optimization for the study of turbulent flow using spectral analysis. Meas. Sci. Technol. 15, 1046-1058.

Ganapathisubramani, B., Longmire, E.K. \& Marusic, I. 2003 Characteristics of vortex packets in turbulent boundary layers. J. Fluid Mech. 478, 35-46.

Guala, M., Hommema, S.E. \& AdRiAn, R.J. 2006 Large-scale and very-large-scale motions in turbulent pipe flow. J. Fluid Mech. 554, 521-542.

Guala, M., Lüthi, B., Liberzon, A., Tsinober, A. \& Kinzelbach, W. 2005 On the evolution of material lines and vorticity in homogeneous turbulence. J. Fluid Mech. 533, 339-359.

Gul, M., Elsinga, G.E. \& WesterweEl, J. 2020 Internal shear layers and edges of uniform momentum zones in a turbulent pipe flow. J. Fluid Mech. 901, A10.

Haller, G. 2005 An objective definition of a vortex. J. Fluid Mech. 525, 1-26.

HeIsel, M. 2020 Organization and scaling of coherent structures in the outer region of high-Reynolds-number turbulent boundary layers. PhD thesis, University of Minnesota.

Heisel, M., Dasari, T., Liu, Y., Hong, J., Coletti, F. \& Guala, M. 2018 The spatial structure of the logarithmic region in very-high-Reynolds-number rough wall turbulent boundary layers. J. Fluid Mech. 857, 704-747.

Heisel, M., Katul, G.G., Chamecki, M. \& Guala, M. $2020 a$ Velocity asymmetry and turbulent transport closure in smooth- and rough-wall boundary layers. Phys. Rev. Fluids 5, 104605.

Heisel, M., De Silva, C.M., Hutchins, N., Marusic, I. \& Guala, M. $2020 b$ On the mixing length eddies and logarithmic mean velocity profile in wall turbulence. J. Fluid Mech. 887, R1.

Herpin, S., Stanislas, M., Foucaut, J.M. \& Coudert, S. 2013 Influence of the Reynolds number on the vortical structures in the logarithmic region of turbulent boundary layers. J. Fluid Mech. 716, 5-50.

Herpin, S., Stanislas, M. \& Soria, J. 2010 The organization of near-wall turbulence: a comparison between boundary layer SPIV data and channel flow DNS data. J. Turbul. 11, N47.

Holzner, M., Guala, M., Lüthi, B., Liberzon, A., Nikitin, N., Kinzelbach, W. \& Tsinober, A. 2010 Viscous tilting and production of vorticity in homogeneous turbulence. Phys. Fluids 22 (6), 061701.

Hunt, J.C.R., Eames, I., Westerweel, J., Davidson, P.A., Voropayev, S., Fernando, J. \& BRAZA, M. 2010 Thin shear layers - the key to turbulence structure? J. Hydro-Environ. Res. 94 (2), 75-82.

Hunt, J.C.R., Ishihara, T., Worth, N.A. \& KanedA, Y. 2014 Thin shear layer structures in high Reynolds number turbulence. Flow Turbul. Combust. 92 (3), 607-649.

Hunt, J.C.R., Wray, A.A. \& Moin, P. 1988 Eddies, Streams, and Convergence Zones in Turbulent Flows. Center for Turbulence Research CTR-S88. Stanford University.

Hutchins, N. \& MARUsic, I. 2007 Evidence of very long meandering features in the logarithmic region of turbulent boundary layers. J. Fluid Mech. 579, 1-28.

HwANG, Y. 2015 Statistical structure of self-sustaining attached eddies in turbulent channel flow. J. Fluid Mech. 767, 254-289.

HWANG, J. \& SUNG, H.J. 2018 Wall-attached structures of velocity fluctuations in a turbulent boundary layer. J. Fluid Mech. 856, 958-983. 


\section{Vortices and shear layers in turbulent boundary layers}

Ishinara, T., Gotoh, T. \& Kaneda, Y. 2009 Study of high-Reynolds number isotropic turbulence by direct numerical simulation. Annu. Rev. Fluid Mech. 41 (1), 165-180.

IshiHARA, T., KANEDA, Y. \& HunT, J.C.R. 2013 Thin shear layers in high Reynolds number turbulence DNS results. Flow Turbul. Combust. 91 (4), 895-929.

JiMÉnEZ, J. 2013 Near-wall turbulence. Phys. Fluids 25, 101302.

JiMÉNEZ, J. 2018 Coherent structures in wall-bounded turbulence. J. Fluid Mech. 842, P1.

Jiménez, J., Wray, A.A., SafFman, P.G. \& Rogallo, R.S. 1993 The structure of intense vorticity in isotropic turbulence. J. Fluid Mech. 255, 65-90.

Kang, S.J., TAnahashi, M. \& MiYauchi, T. 2007 Dynamics of fine scale eddy clusters in turbulent channel flows. J. Turbul. 8, N52.

VON KÁRMÁN, T. 1930 Mechanische ähnlichkeit und turbulenz. Gött. Nachr. 5, 58-76.

KIM, K.C. \& AdRIAN, R.J. 1999 Very large-scale motion in the outer layer. Phys. Fluids 11 (2), 417-422.

KLEWICKI, J.C. 2010 Reynolds number dependence, scaling, and dynamics of turbulent boundary layers. Trans. ASME J. Fluids Engng 132 (9), 094001.

KLEWICKI, J.C. 2013 A description of turbulent wall-flow vorticity consistent with mean dynamics. J. Fluid Mech. 737, 176-204.

KLEWICKI, J.C. \& HiRSCHI, C.R. 2004 Flow field properties local to near-wall shear layers in a low Reynolds number turbulent boundary layer. Phys. Fluids 16 (11), 4163-4176.

Kolmogorov, A.N. 1941 The local structure of turbulence in an incompressible viscous fluid for very large Reynolds numbers. Dokl. Akad. Nauk SSSR 30, 299-303.

Laskari, A., DE KAT, R., Hearst, R.J. \& Ganapathisubramani, B. 2018 Time evolution of uniform momentum zones in a turbulent boundary layer. J. Fluid Mech. 842, 554-590.

LASKARI, A. \& MCKEON, B.J. 2021 Temporal characteristics of the probability density function of velocity in wall-bounded turbulent flows. J. Fluid Mech. 913, A6.

LozAno-Durán, A. \& BAE, H.J. 2019 Characteristic scales of Townsend's wall-attached eddies. J. Fluid Mech. 868, 698-725.

Lozano-Durán, A., Flores, O. \& Jiménez, J. 2012 The three-dimensional structure of momentum transfer in turbulent channels. J. Fluid Mech. 694, 100-130.

LOZANO-DuRÁN, A. \& JimÉnEZ, J. 2014 Time-resolved evolution of coherent structures in turbulent channels: characterization of eddies and cascades. J. Fluid Mech. 759, 432-471.

Marusic, I., McKeon, B.J., Monkewitz, P.A., Nagib, H.M., Smits, A.J. \& Sreenivasan, K.R. 2010 Wall-bounded turbulent flows at high Reynolds number: recent advances and key issues. Phys. Fluids $22(6), 065103$.

MARUSiC, I. \& Monty, J.P. 2019 Attached eddy model of wall turbulence. Annu. Rev. Fluid Mech. 51, 49-74.

McKeon, B.J. \& SReEnivasan, K.R. 2007 Introduction: scaling and structure in high Reynolds number wall-bounded flows. Phil. Trans. R. Soc. A 365 (1852), 635-646.

MEINHART, C.D. \& ADRIAN, R.J. 1995 On the existence of uniform momentum zones in a turbulent boundary layer. Phy. Fluids 7 (4), 694-696.

MoISY, F. \& JIMÉNEZ, J. 2004 Geometry and clustering of intense structures in isotropic turbulence. J. Fluid Mech. 513, 111-133.

Montemuro, B., White, C.M., Klewicki, J.C. \& Chini, G.P. 2020 A self-sustaining process theory for uniform momentum zones and internal shear layers in high reynolds number shear flows. J. Fluid Mech. 901, A28.

Morishita, K., Ishihara, T. \& KanedA, Y. 2019 Length scales in turbulent channel flow. J. Phys. Soc. Japan 88, 064401.

Morris, S.C., Stolpa, S.R., Slaboch, R.E. \& Klewicki, J.C. 2007 Near-surface particle image velocimetry measurements in a transitionally rough-wall atmospheric boundary layer. J. Fluid Mech. 580, 319-338.

OSEEN, C.W. 1912 Über die wirbelbewegung in einer reibenden flüssigkeit. Ark. Mat. Astro. Fys. 7, 14-26.

Pope, S.B. 2000 Turbulent Flows, 1st edn. Cambridge University Press.

Priyadarshana, P.J.A., Klewicki, J.C., Treat, S. \& Foss, J.F. 2007 Statistical structure of turbulent-boundary-layer velocity-vorticity products at high and low Reynolds numbers. J. Fluid Mech. 570, 307-346.

REYNOLDS, O. 1883 An experimental investigation of the circumstances which determine whether the motion of water shall be direct or sinuous, and of the law of resistance in parallel channels. Phil. Trans. R. Soc. 174, 935-982.

Saddoughi, S.G. \& Veeravalli, S.V. 1994 Local isotropy in turbulent boundary layers at high Reynolds number. J. Fluid Mech. 268, 333-372. 


\section{Heisel, C.M. de Silva, N. Hutchins, I. Marusic and M. Guala}

SAFFMAN, P.G. 1968 Lectures on homogeneous turbulence. In Topics in Non-linear Physics (ed. N. Zabusky), pp. 485-614. Springer.

Saxton-Fox, T. \& MCKeOn, B.J. 2017 Coherent structures, uniform momentum zones and the streamwise energy spectrum in wall-bounded turbulent flows. J. Fluid Mech. 826, R6.

SHE, Z.S., JACKSON, E. \& ORSZAG, S.A. 1990 Intermittent vortex structures in homogeneous isotropic turbulence. Nature 344 (3), 226-228.

She, Z.S. \& Leveque, E. 1994 Universal scaling laws in fully developed turbulence. Phys. Rev. Lett. 72 (3), 336-339.

Sillero, J.A., JiméneZ, J. \& Moser, R.D. 2013 One-point statistics for turbulent wall-bounded flows at Reynolds numbers up to $\delta^{+} \approx 2000$. Phys. Fluids 25, 105102.

DA Silva, C.B., Hunt, J.C.R., EAmes, I. \& WesterweEl, J. 2014 Interfacial layers between regions of different turbulence intensity. Annu. Rev. Fluid Mech. 46, 567-590.

De Silva, C.M., Hutchins, N. \& Marusic, I. 2016 Uniform momentum zones in turbulent boundary layers. J. Fluid Mech. 786, 309-331.

De Silva, C.M., Philip, J., Hutchins, N. \& Marusic, I. 2017 Interfaces of uniform momentum zones in turbulent boundary layers. J. Fluid Mech. 820, 451-478.

DA Silva, C.B. \& TAVEIRA, R. 2010 The thickness of the turbulent/nonturbulent interface is equal to the radius of the large vorticity structures near the edge of the shear layer. Phys. Fluids 22 (12), 021702.

Smits, A.J., McKeon, B.J. \& Marusic, I. 2011 High-Reynolds number wall turbulence. Annu. Rev. Fluid Mech. 43 (1), 353-375.

SQUIRE, D.T. 2017 The structure and scaling of rough-wall turbulent boundary layers. PhD thesis, University of Melbourne.

Squire, D.T., Morrill-Winter, C., Hutchins, N., Marusic, I., Schultz, M.P. \& Klewicki, J.C. 2016 Smooth- and rough-wall boundary layer structure from high spatial range particle image velocimetry. Phys. Rev. Fluids 1 (6), 064402.

Sreenivasan, K.R. \& Antonia, R.A. 1997 The phenomenology of small-scale turbulence. Annu. Rev. Fluid Mech. 29, 435-472.

Tanahashi, M., Kang, S.J., Miyamoto, T., Shiokawa, S. \& Miyauchi, T. 2004 Scaling law of fine scale eddies in turbulent channel flows up to $R e_{\tau}=800$. Intl J. Heat Fluid Flow 25 (3), 331-340.

TAYLOR, G.I. 1935 Statistical theory of turbulence: parts I-III. Proc. R. Soc. A 151 (873), 421-464.

Townsend, A.A. 1961 Equilibrium layers and wall turbulence. J. Fluid Mech. 11 (1), 97-120.

Townsend, A.A. 1976 The Structure of Turbulent Shear Flow, 2nd edn. Cambridge University Press.

TSINOBER, A. 2001 An Informal Introduction to Turbulence. Springer.

Wei, L., Elsinga, G.E., Brethouwer, G., Schlatter, P. \& Johansson, A. V. 2014 Universality and scaling phenomenology of small-scale turbulence in wall-bounded flows. Phys. Fluids 26 (3), 035107.

Wu, Y. \& CHRistensen, K.T. 2006 Population trends of spanwise vortices in wall turbulence. J. Fluid Mech. 568, 55-76. 